\title{
CRISPR-based diagnostics
}

\author{
Michael M. Kaminski1,2, Omar O. Abudayyeh ${ }^{3,4}$, Jonathan S. Gootenberg ${ }^{3,4}$, Feng Zhang ${ }^{3,4,5,6,7,8}$ and

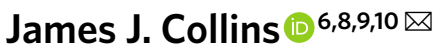

The accurate and timely diagnosis of disease is a prerequisite for efficient therapeutic intervention and epidemiological surveillance. Diagnostics based on the detection of nucleic acids are among the most sensitive and specific, yet most such assays require costly equipment and trained personnel. Recent developments in diagnostic technologies, in particular those leveraging clustered regularly interspaced short palindromic repeats (CRISPR), aim to enable accurate testing at home, at the point of care and in the field. In this Review, we provide a rundown of the rapidly expanding toolbox for CRISPR-based diagnostics, in particular the various assays, preamplification strategies and readouts, and highlight their main applications in the sensing of a wide range of molecular targets relevant to human health.

T he fast and accurate diagnosis of a disease is central to effective treatment and to the prevention of long-term sequelae ${ }^{1}$. Nucleic-acid-based biomarkers associated with disease are essential for diagnostics because DNA and RNA can be amplified from trace amounts, which enables their highly specific detection via the pairing of complementary nucleotides. In fact, nucleic-acid-based diagnostics have become the gold standard for various acute and chronic conditions, especially those caused by infectious diseases ${ }^{2}$. During infectious-disease outbreaks, as most recently experienced with the coronavirus disease 2019 (COVID19) pandemic, fast and precise nucleic-acid-based testing is vital for effective disease control ${ }^{3,4}$. The detection of nucleic acid biomarkers is also critical for agriculture and food safety, for environmental monitoring and in the sensing of biological warfare agents.

Nucleic acid-based diagnostics relying on the quantitative polymerase chain reaction (qPCR) or on sequencing have been widely adopted, and are frequently used in clinical laboratories. The versatility, robustness and sensitivity of PCR have made this technology the most commonly used for the detection of DNA and RNA biomarkers. In fact, PCR is the gold-standard technique for most nucleic-acid-based diagnostics. However, the costs of reagents for PCR are high, and the technique requires sophisticated laboratory equipment and trained personnel ${ }^{5}$. Although isothermal nucleic acid amplification circumvents the need for thermal cyclers, non-specific amplification can result in lower detection specificity ${ }^{6}$. The specificity can be improved through additional readouts, in particular by fluorescent probes ${ }^{7}$, oligo strand-displacement probes ${ }^{8}$ or molecular beacons?. However, there is a need for technologies that combine the ease of use and cost efficiency of isothermal amplification with the diagnostic accuracy of PCR. Ideally, such next-generation diagnostics should also have single-nucleotide specificity, which is integral to the detection of mutations conferring resistance against antibiotics ${ }^{10}$ or antiviral drugs ${ }^{11}$.

Clustered regularly interspaced short palindromic repeats (CRISPR)-based diagnostics have the potential to fulfil these unmet needs. CRISPR systems are a fundamental part of a microbial adaptive immune system ${ }^{12}$ that recognizes foreign nucleic acids on the basis of their sequence to subsequently eliminate them by means of endonuclease activity associated with the CRISPR-associated (Cas) enzyme. Although there are diverse CRISPR-Cas systems among the different species of archaea and bacteria ${ }^{13}$, these systems are connected by their dependence on CRISPR RNA (crRNA), which guides Cas proteins to recognize and cleave nucleic acid targets. The crRNA can be programmed towards a specific DNA or RNA region of interest through hybridization to a complementary sequence, which in some systems is restricted to the proximity of a protospacer adjacent motif (PAM) or protospacer flanking sequence ${ }^{14}$. So far, CRISPR-Cas systems have been repurposed for a variety of applications, including the targeted editing of genomes ${ }^{15}$, epigenomes $^{16}$ and transcriptomes ${ }^{17}$, the bioimaging of nucleic acids ${ }^{18}$, the recording of cellular events ${ }^{19}$ and the detection of nucleic acids. Overall, the fast-evolving area of CRISPR-based diagnostics builds on the specificity, programmability and ease of use of CRISPR technology, and aims to create nucleic-acid-based point-of-care (POC) diagnostic tests for use in routine clinical care.

In this Review, we outline how the properties of different Cas enzymes have been leveraged for diagnostic assays, discuss different preamplification strategies (which today form part of most CRISPR-based diagnostic assays), examine approaches for quantification and multiplexing, highlight technologies that combine CRISPR-based target enrichment with sequencing, and review the optimization of CRISPR-based diagnostics for POC applications, with a focus on assay readouts and sample preparation. We also provide an overview of the emerging biomedical applications of the technology, and discuss open challenges and opportunities.

\section{CRISPR-based methods for disease diagnosis}

Since their initial discovery, the number of different CRISPR-Cas systems has expanded rapidly ${ }^{13,20,21}$. Currently, CRISPR-Cas systems can be divided, according to evolutionary relationships, into two classes, six types and several subtypes ${ }^{20}$. The classes of CRISPRCas system are defined by the nature of the ribonucleoprotein effector complex: class 1 systems are characterized by a complex of multiple effector proteins, and class 2 systems encompass a single

'Berlin Institute for Medical Systems Biology, Max Delbrück Center for Molecular Medicine, Berlin, Germany. ${ }^{2}$ Department of Nephrology and Medical Intensive Care, Charité-Universitätsmedizin Berlin, Berlin, Germany. ${ }^{3}$ McGovern Institute for Brain Research at MIT, Cambridge, MA, USA. ${ }^{4}$ Massachusetts Consortium for Pathogen Readiness, Boston, MA, USA. ${ }^{5}$ Howard Hughes Medical Institute, Cambridge, MA, USA. ${ }^{6}$ Broad Institute of MIT and Harvard, Cambridge, MA, USA. 'Department of Brain and Cognitive Sciences, MIT, Cambridge, MA, USA. ${ }^{8}$ Department of Biological Engineering, MIT, Cambridge, MA, USA. ${ }^{9}$ Institute for Medical Engineering and Science, MIT, Cambridge, MA, USA. ${ }^{10}$ Wyss Institute for Biologically Inspired Engineering, Harvard University, Boston, MA, USA. 凶e-mail: jimjc@mit.edu 
Table 1 | Design principles for crRNAs used in CRISPR-based diagnostics

\begin{tabular}{|llll} 
Enzyme & $\begin{array}{l}\text { Spacer } \\
\text { length (bp) }\end{array}$ & $\begin{array}{l}\text { Direct-repeat } \\
\text { length (bp) }\end{array}$ & $\begin{array}{l}\text { Direct-repeat } \\
\text { orientation }\end{array}$ \\
\hline AsCas12a $\left(\right.$ ref. $\left.^{22}\right)$ & 20 & 20 & $5^{\prime}$ \\
\hline LbCas12a $\left(\right.$ ref. $\left.^{36}\right)$ & 20 & 21 & $5^{\prime}$ \\
\hline LbaCas13a $\left(\right.$ ref. $\left.^{34}\right)$ & 28 & 35 & $5^{\prime}$ \\
\hline LbuCas13a $\left(\right.$ ref. $\left.^{22}\right)$ & 20 & 31 & $5^{\prime}$ \\
\hline LwaCas13a $\left(\right.$ ref. $\left.^{22}\right)$ & 28 & 36 & $5^{\prime}$ \\
\hline CcaCas13b $\left(\right.$ ref. $\left.^{22}\right)$ & 30 & 36 & $3^{\prime}$ \\
\hline PsmCas13b (ref. $\left.{ }^{22}\right)$ & 30 & 36 & $3^{\prime}$ \\
\hline
\end{tabular}

bp, base pair.

crRNA-binding protein. The design of crRNAs for the different effector proteins used in CRISPR diagnostics follow the same principles as those of other CRISPR applications, and are summarized in Table 1. Among the diverse CRISPR systems, class 2 systems have primarily been applied for diagnostics, as these systems are simpler to reconstitute. They include enzymes with collateral activity, which serves as the backbone of many CRISPR-based diagnostic assays (Table 2). Class 1 systems (such as the type III effector nuclease Csm6 or Cas10) have also been engineered for diagnostics, either in combination with components of the class 2 system or with the native type III complex ${ }^{22,23}$.

The first CRISPR-based diagnostic methods that were developed largely used Cas9 variants, which recognize double-stranded DNA (dsDNA). The main principles of the many different Cas9-based approaches for sensing DNA that have been reported include guide-directed reconstitution of split proteins by catalytically inactive Cas9 partners ${ }^{24}$, Cas9-based destruction of PAM-containing sites $^{25,26}$ and Cas9-induced unwinding of the non-targeted DNA strand as a targeting site for isothermal amplification ${ }^{27}$. The Cas9-based method referred to as NASBACC (for nucleic acid sequence-based amplification (NASBA)-CRISPR cleavage) ${ }^{25}$ combines nucleic acid sequence-based amplification ${ }^{28}$ for the isothermal preamplification of targets, Cas9 cleavage for PAM-dependent target detection and a toehold sensor for the readout (Fig. 1, left). In brief, a toehold trigger is attached to a NASBA-amplified RNA fragment through reverse transcription. If a PAM sequence is present in the RNA fragment, Cas9-mediated cleavage leads to a truncated RNA without the trigger sequence. In the absence of the PAM sequence, the trigger containing full-length RNA activates the toehold switch, as indicated by a colour change. By sensing strain-specific PAM sites, the method allows for viral-lineage discrimination, as shown for the detection of the Zika virus (ZIKV) in infected monkey plasma at concentrations in the low femtomolar range $^{25}$. Reported most recently, a Cas9-based method referred to as LEOPARD (for leveraging engineered tracrRNAs and on-target DNAs for parallel RNA detection) enables the multiplexed detection of different RNA sequences with single-nucleotide specificity ${ }^{29}$. The method is based on the observation that trans-activating crRNAs (tracrRNAs) - which in type II systems are necessary for the formation of Cas9-crRNA complexes-hybridize to cellular RNAs, making 'non-canonical' crRNAs. By reprogramming tracrRNAs to bind cellular transcripts of interest while allowing for the formation of Cas9-crRNA complexes, the resulting non-canonical crRNAs enable the guiding of Cas9 to a DNA target.

The main difference between CRISPR type II (Cas9) systems and type V (Cas12) and type VI (Cas13) systems is the ability of the latter two systems to trigger non-specific collateral cleavage (trans cleavage) on target recognition. Collateral activity involves the cleavage of non-targeted single-stranded DNA (ssDNA; Cas12) or single-stranded RNA (ssRNA; Cas13) in solution, which enables the sensing of nucleic acids through signal amplification and allows for various readouts through the addition of functionalized reporter nucleic acids, which are cleaved by collateral activity. The CRISPR-Cas type VI (Cas13) family of enzymes has a size range of $~ 900-1,300$ amino acids, detects ssRNA in cis conformation and shows collateral trans-cleavage activity against ssRNA in vitro ${ }^{30,31}$. In the Cas13-based assay SHERLOCK (for specific high-sensitivity enzymatic reporter unlocking $)^{32}$, DNA or RNA is first isothermally amplified with recombinase polymerase amplification (RPA) ${ }^{33}$ or reverse transcription RPA (RT-RPA), respectively, using a forward primer that adds a T7 promoter to the amplicon (Fig. 1, right). This promoter allows for RNA transcription of the target, which is then recognized and bound by a complex of Cas13a from Leptotrichia wadeii (LwaCas13a) and a crRNA that includes a complementary sequence to the target. The activated Cas13 will then cleave both the on-target RNA by cis cleavage and, in a target-dependent manner, the ssRNA reporter molecules by collateral trans cleavage. The ssRNA reporter molecules consist of a fluorophore and a quencher joined together by a short RNA oligomer, which, once cleaved, allows for the separation of the fluorophore from the quencher, resulting in fluorescence. These processes enable the detection of viral RNA, bacterial DNA, human single-nucleotide polymorphisms (SNPs) and cancer-associated mutations with attomolar $\left(10^{-18} \mathrm{M}\right)$ sensitivity. Version two of the assay (SHERLOCKv2) enabled the quantitative multiplexed sensing of nucleic acids and the target detection at zeptomolar $\left(10^{-21} \mathrm{M}\right)$ concentrations, and introduced a lateral-flow readout based on an immunochromatographic assay, where cleaved reporter molecules are detected through antibody-conjugated gold nanoparticles on a paper strip ${ }^{22,34}$.

Cas12 enzymes that have been used in CRISPR-based diagnostics target dsDNA and ssDNA, require a PAM site in the target region for dsDNA cleavage and collaterally cleave ssDNA ${ }^{35}$. One of the first Cas12-based detection methods was reported in 2018 and referred to as DETECTR (for DNA endonuclease-targeted CRISPR trans reporter; Fig. 1, right) ${ }^{36}$. In this method, Cas12a from Lachnospiraceae bacterium (LbCas12a) or other organisms is guided to dsDNA targets by a complementary crRNA, triggering collateral cleavage of short ssDNA reporters carrying a fluorophore and a quencher. Similar to SHERLOCK, target recognition and reporter cleavage lead to the separation of the quencher from the fluorophore, which generates a fluorescence signal. DETECTR reached attomolar sensitivity when combined with RPA preamplification. Other Cas12-based techniques include HOLMES (for one-hour low-cost multipurpose highly efficient system) ) $^{37,38}$, which employs PCR as preamplification together with LbCas12a, and HOLMESv2 ${ }^{39}$, which uses loop-mediated isothermal amplification (LAMP $)^{40}$ combined with a thermostable Cas12b from Alicyclobacillus acidoterrestris (AacCas12b) in a one-pot reaction. Both HOLMES and HOLMESv2 showed a limit of detection (LOD) around $10 \mathrm{aM}$. Similarly, Cas12f targets dsDNA and ssDNA, but enables better discrimination of SNPs in ssDNA than Cas $12 \mathrm{a}^{41}$.

\section{Preamplification-free diagnostics}

When using Cas enzymes without upstream preamplification of the target, most CRISPR-based diagnostics have a reported LOD in the picomolar range ${ }^{32,42}$. This LOD allows for target detection when there is a relatively high concentration of DNA or RNA in the sample. For example, severe acute respiratory syndrome coronavirus 2 (SARS-CoV-2) is apparently shed at high concentration in the early phase of infection $\left(\sim 6.76 \times 10^{5} \text { copies per swab }\right)^{43}$, allowing preamplification-free detection ${ }^{44}$. In addition to highly concentrated microbial samples, human genomic DNA (gDNA) ${ }^{45}$ and highly expressed human messenger $\mathrm{RNAs}^{30}$ and microRNAs (miRNAs) ${ }^{46}$ have been reported to be amenable to detection without preamplification. 
Table 2 | Characteristics of reported CRISPR-based diagnostics

\begin{tabular}{|c|c|c|c|c|c|c|c|c|c|}
\hline Name & Enzyme & Preamplification & Assay time ${ }^{a}(\mathrm{~min})$ & $\begin{array}{l}\text { Sample } \\
\text { preparation }\end{array}$ & Readout & Applications & $\operatorname{LOD}^{c}\left(\mathrm{moll}^{-1}\right)$ & $\begin{array}{l}\text { LODc }^{c} \\
(\text { copies } \\
\text { per } \mathrm{ml})^{3}\end{array}$ & References \\
\hline \multicolumn{10}{|l|}{ CRISPR type II } \\
\hline NASBACC ${ }^{b}$ & Cas9 & NASBA & $120-360$ (one pot) & $\begin{array}{l}\text { Column-based or } \\
\text { crude extraction }\end{array}$ & Colometry & $\begin{array}{l}\text { Discrimination between } \\
\text { African and American ZIKV }\end{array}$ & $1.0 \times 10^{-15}$ & $6.0 \times 10^{5}$ & 25 \\
\hline CRISPR-Chip & Cas9 & - & 15 & Column-based & Electrochemical & $\begin{array}{l}\text { Detection of gDNA from cell } \\
\text { lines and DMD patients }\end{array}$ & $2.3 \times 10^{-15}$ & $1.4 \times 10^{6}$ & 45 \\
\hline CRISDA & $\begin{array}{l}\text { Cas9 } \\
\text { nickase }\end{array}$ & SDA & 90 & Column-based & Fluorescence & $\begin{array}{l}\text { Detection of gDNA; } \\
\text { breast-cancer-associated } \\
\text { SNPs in cell lines }\end{array}$ & $2.5 \times 10^{-19}$ & $1.5 \times 10^{2}$ & 27 \\
\hline FLASH & Cas9 & PCR & NS & Column-based & NGS & $\begin{array}{l}\text { Detection of gDNA; } \\
\text { antimicrobial resistance } \\
\text { genes in clinical samples }\end{array}$ & $1.9 \times 10^{-18}$ & $1.1 \times 10^{3}$ & 71 \\
\hline CAS-EXPAR & Cas9 & EXPAR & 60 & $\begin{array}{l}\text { Chemical (phase } \\
\text { separation) }\end{array}$ & Fluorescence & $\begin{array}{l}\text { Sensing of methylated DNA; } \\
\text { L. monocytogenes mRNA }\end{array}$ & $8.2 \times 10^{-19}$ & $4.9 \times 10^{2}$ & 91 \\
\hline Cas9nAR & $\begin{array}{l}\text { Cas9 } \\
\text { nickase }\end{array}$ & $\begin{array}{l}\text { Strand- } \\
\text { displacing DNA } \\
\text { polymerase }\end{array}$ & 60 & Column-based & Fluorescence & $\begin{array}{l}\text { Detection of bacteria (S. } \\
\text { typhimurium, E. coli, M. } \\
\text { smegmatis, S. erythraea); } \\
\text { detection of KRAS SNPs in } \\
\text { cell lines }\end{array}$ & $1.7 \times 10^{-19}$ & $1.0 \times 10^{2}$ & 111 \\
\hline \multicolumn{10}{|l|}{ CRISPR type V } \\
\hline DETECTR & Cas12a & RPA & $\begin{array}{l}10 \text { (RPA) and } \\
60-120 \text { (CRISPR) }\end{array}$ & Crude extraction & Fluorescence & $\begin{array}{l}\text { Detection of HPV16 and } \\
\text { HPV18 in human samples }\end{array}$ & $1.0 \times 10^{-18}$ & $6.0 \times 10^{2}$ & 36 \\
\hline Cas14-DETECTR & $\begin{array}{l}\text { Cas14 } \\
\text { (Cas12f) }\end{array}$ & PCR & $\begin{array}{l}\text { NS (PCR) and } \\
120 \text { (CRISPR) }\end{array}$ & Crude extraction & Fluorescence & $\begin{array}{l}\text { Detection of HERC2 SNPs in } \\
\text { human samples }\end{array}$ & n.s. & $6.0 \times 10^{3}$ & 41 \\
\hline HOLMES & Cas12a & PCR & $\begin{array}{l}88 \text { (PCR) and } 15 \\
\text { (CRISPR) }\end{array}$ & Column-based & Fluorescence & $\begin{array}{l}\text { SNP discrimination in cell } \\
\text { lines and human samples; } \\
\text { detection of viruses } \\
\text { (PRV, JEV); virus-strain } \\
\text { discrimination }\end{array}$ & $1.0 \times 10^{-17}$ & $6.6 \times 10^{3}$ & 37,38 \\
\hline CRISPR-materials & Cas12a & RPA & $\begin{array}{l}40 \text { (RPA) and } 240 \\
\text { (CRISPR) }\end{array}$ & Synthetic targets & $\begin{array}{l}\text { Fluorescence or } \\
\mu \text { PAD (visual and } \\
\text { electronic) }\end{array}$ & $\begin{array}{l}\text { Detection of EBOV synthetic } \\
\text { RNA }\end{array}$ & $1.0 \times 10^{-17}$ & $6.6 \times 10^{3}$ & 79,80 \\
\hline CDetection & Cas12b & RPA & $\begin{array}{l}10 \text { (RPA) and } \\
60-180 \text { (CRISPR) }\end{array}$ & $\begin{array}{l}\text { Synthetic targets } \\
\text { or crude extraction }\end{array}$ & Fluorescence & $\begin{array}{l}\text { Detection of HPV16; human } \\
\text { ABO blood genotyping; } \\
\text { BRCA1 and TP53 SNPs }\end{array}$ & $1.0 \times 10^{-18}$ & $6.0 \times 10^{2}$ & 112 \\
\hline HOLMESV2 & Cas12b & LAMP & $\begin{array}{l}40 \text { (LAMP) and } 35 \\
\text { (CRISPR) or } 120 \\
\text { (one pot) }\end{array}$ & NS & Fluorescence & $\begin{array}{l}\text { SNP discrimination in cell } \\
\text { lines; RNA virus detection } \\
\text { (JEV); human mRNA and } \\
\text { circular RNA detection; DNA } \\
\text { methylation }\end{array}$ & $1.0 \times 10^{-17}$ & $6.0 \times 10^{3}$ & 39 \\
\hline E-CRISPR & Cas12a & - & $30-180$ & $\begin{array}{l}\text { Synthetic targets } \\
\text { (nucleic acids) }\end{array}$ & Electrochemical & $\begin{array}{l}\text { Detection of viruses (HPV16, } \\
\text { PB19) and protein (TGF-B1) }\end{array}$ & $5.0 \times 10^{-11}$ & $3.0 \times 10^{10}$ & 77 \\
\hline \multicolumn{10}{|l|}{ CRISPR type VI } \\
\hline- & Cas13 & - & NS & NS & Fluorescence & $\begin{array}{l}\text { Detection of human mRNA; } \\
\text { detection of bacteriophage } \\
\lambda \text {-RNA }\end{array}$ & $1.0 \times 10^{-12}$ & $6.0 \times 10^{8}$ & 30,31 \\
\hline SHERLOCK & Cas13 & NASBA or RPA & $\begin{array}{l}132(\text { NASBA }) \text { or } \\
120 \text { (RPA) and } \\
60-180 \text { (CRISPR) }\end{array}$ & $\begin{array}{l}\text { Column-based or } \\
\text { crude extraction }\end{array}$ & Fluorescence & $\begin{array}{l}\text { Detection of viruses (ZIKV, } \\
\text { DENV) and bacteria (E. coli, } \\
\text { K. pneumoniae, P. aeruginosa, } \\
\text { M. tuberculosis, S. aureus); } \\
\text { discrimination between virus } \\
\text { strains; detection of SNPs }\end{array}$ & $2.0 \times 10^{-18}$ & $1.2 \times 10^{3}$ & 32 \\
\hline SHERLOCKv $2^{\mathrm{b}}$ & Cas13 & RPA & $\begin{array}{l}60 \text { (RPA) and } \\
60-180 \text { (CRISPR) } \\
\text { or } 60-180 \text { (one } \\
\text { pot) }\end{array}$ & $\begin{array}{l}\text { Column-based or } \\
\text { crude extraction }\end{array}$ & $\begin{array}{l}\text { Fluorescence or } \\
\text { lateral flow }\end{array}$ & $\begin{array}{l}\text { Detection of viruses (ZIKV, } \\
\text { DENV) and bacteria ( } P \text {. } \\
\text { aeruginosa, S. aureus); } \\
\text { discrimination between virus } \\
\text { strains; detection of SNPS }\end{array}$ & $8.0 \times 10^{-21}$ & 4.8 & 22,34 \\
\hline SHINE & Cas13 & RPA & 50 (one pot) & Crude extraction & $\begin{array}{l}\text { Fluorescence or } \\
\text { lateral flow }\end{array}$ & Detection of SARS-CoV-2 & $8.3 \times 10^{-18}$ & $5.0 \times 10^{3}$ & 62 \\
\hline STOPCovid & Cas12b & LAMP & 60 (one pot) & Crude extraction & $\begin{array}{l}\text { Fluorescence or } \\
\text { lateral flow }\end{array}$ & Detection of SARS-CoV-2 & $3.3 \times 10^{-18}$ & $2.0 \times 10^{3}$ & 63 \\
\hline CARMEN & Cas13 & PCR or RPA & $\begin{array}{l}20 \text { (RPA) and } 180 \\
\text { (CRISPR) }\end{array}$ & Column-based & Fluorescence & $\begin{array}{l}\text { Detection of } 169 \text { viruses; } \\
\text { subtyping of influenza A } \\
\text { strains; detection of HIV } \\
\text { drug-resistant mutations }\end{array}$ & $9 \times 10^{-19}$ & $5.4 \times 10^{2}$ & 67 \\
\hline APC-Cas & Cas13 & $\begin{array}{l}\text { Allosteric- } \\
\text { probe-initiated } \\
\text { amplification } \\
\text { with DNA } \\
\text { polymerase }\end{array}$ & $\begin{array}{l}110 \text { (APC) and } \\
30 \text { (CRISPR) }\end{array}$ & None & Fluorescence & Detection of S. enteritidis & $\begin{array}{l}\text { One colony- } \\
\text { forming unit }\end{array}$ & - & 92 \\
\hline
\end{tabular}


Table 2 | Characteristics of reported CRISPR-based diagnostics (Continued)

\begin{tabular}{|c|c|c|c|c|c|c|c|c|c|}
\hline Name & Enzyme & Preamplification & Assay time ${ }^{a}(\mathrm{~min})$ & $\begin{array}{l}\text { Sample } \\
\text { preparation }\end{array}$ & Readout & Applications & $\operatorname{LOD}^{c}\left(\mathrm{moll}^{-1}\right)$ & $\begin{array}{l}\text { LOD }^{c} \\
\text { (copies } \\
\text { per } \mathrm{ml})^{3}\end{array}$ & References \\
\hline & Cas13 & - & $<240$ & Column-based & Electrochemical & $\begin{array}{l}\text { Detection of microRNAs } \\
\text { (miR-19b and miR-20a) }\end{array}$ & $1 \times 10^{-11}$ & $6.0 \times 10^{9}$ & 46 \\
\hline PECL-CRISPR & Cas13 & EXPAR & $\begin{array}{l}30 \text { (CRISPR), } 30 \\
\text { (phosphorylation } \\
\text { of pre-trigger), } 30 \\
\text { (EXPAR) }\end{array}$ & Column-based & Electrochemiluminescence & $\begin{array}{l}\text { Detection of microRNAs (miR- } \\
\text { 17, let-7 family miRNAs) }\end{array}$ & $1.0 \times 10^{-15}$ & $6.0 \times 10^{5}$ & 78 \\
\hline
\end{tabular}

NS, not specified; APC-Cas, allosteric probe-initiated catalysis and CRISPR-Cas13a system; BRCA1, breast cancer 1 gene; circRNA, circular RNA; Cas9nAR, Cas9 nickase-based amplification reaction; CRISDA, CRISPR-Cas9-triggered nicking endonuclease-mediated strand-displacement amplification; DENV, dengue virus; DMD, Duchenne muscular dystrophy; EBOV, Ebola virus; E. coli, Escherichia coli; HERC2, HECT and RLD domain containing E3 ubiquitin protein ligase 2 gene; HPV, human papillomavirus; JEV, Japanese encephalitis virus; K. pneumoniae, Klebsiella pneumoniae; KRAS, KRAS proto-oncogene GTPase; M. smegmatis, Mycobacterium smegmatis; M. tuberculosis, Mycobacterium tuberculosis; PECL, portable electrochemiluminescence chip; P. aeruginosa, Pseudomonas aeruginosa; PB19, parvovirus B19; PRV, pseudorabies virus; S. erythraea, Saccharopolyspora erythraea; S. aureus, Staphylococcus aureus; S. enteritidis, Salmonella enteritidis; S. typhimurium, Salmonella typhimurium; TP53, tumour protein P53 gene. ${ }^{a}$ Assay time indicates the approximate incubation time most frequently used in the referred study (different assay times can be reported, depending on the intended sensitivity and the readout). ${ }^{\mathrm{b} P O C}$ compatibility indicates whether the entire assay as reported-including sample preparation (that is, crude extraction) and readout-can be performed at POC or in the field with minimal equipment. 'Limits of detection cannot always be directly compared across studies, in particular because some studies did not report how the LOD was determined, or reported the target concentration either in the transport media of the sample or in the final reaction. The LODs shown here reflect the optimal LODs reported. In general, LODs depend on the type of input material (raw or synthetic), type of readout and incubation time.

To increase the sensitivity of preamplification-free CRISPR-based diagnostics, LwaCas13a has been combined with the CRISPR type III RNA nuclease Csm6 ${ }^{22}$. The nuclease activity of Csm6 is activated by cyclic oligoadenylates $\left(2^{\prime}, 3^{\prime} \text {-cyclic phosphate groups }\right)^{47,48}$. Because the collateral-cleavage activity of both LwaCas13a and PsmCas13b (from Prevotella sp. MA2016) generate products with hydroxylated $5^{\prime}$ ends and $2^{\prime}, 3^{\prime}$-cyclic phosphate ends, it was hypothesized that the collateral activity of Cas 13 could generate Csm6 activators, thereby enabling amplified signal detection. In contrast to the cleavage preferences of LwaCas13a for the UU and AU two-base motifs, it was found that Csm6 from Enterococcus italicus (EiCsm6), Lactobacillus salivarius (LsCsm6) and Thermus thermophilus (TtCsm6) had strong cleavage preferences for A-rich and $\mathrm{C}$-rich reporters, allowing for the cleavage activity of LwaCas13a and Csm6 to be independently measured in different channels and for the further design of 'protected' RNA activators. These activators were designed to contain a poly(A) Csm6 activator followed by a $\operatorname{poly}(\mathrm{U})$ stretch, on the basis of the rationale that the collateral cleavage of LwaCas13a would only degrade the uridines, thereby generating hexadenylates with $2^{\prime}, 3^{\prime}$-cyclic phosphate ends and activating Csm6. Activated Csm6 would in turn cleave additional reporter molecules, freeing up quenched fluorophores and thereby increasing the signal. This design led to a 3.5 -fold increase in signal sensitivity when compared with Cas13a alone and when reading Csm6-mediated fluorescence and Cas13a-mediated fluorescence in the same channel. Similarly, the generation of cyclic oligoadenylates from the native Cas 10 enzyme in type III systems has been used for detection of SARS-CoV-2 genomes ${ }^{23}$.

More strategies that increase the sensitivity of preamplificationfree CRISPR-based diagnostics are needed. This could include the Cas-enzyme-mediated degradation of aptamers or of enzyme-inhibiting nucleic acid linkers (such as calf intestinal alkaline phosphatase or luciferase ${ }^{49}$ ) that can then be detected via colorimetric or luminescence methods.

\section{Preamplification strategies}

In contrast to applications that are amenable to preamplification-free CRISPR-based diagnostics, most clinical settings require the ability to sense nucleic acid concentrations below the picomolar range $\mathrm{e}^{50}$. An example is the sensing of human immunodeficiency virus (HIV) or hepatitis $\mathrm{C}$ virus (HCV) load under antiviral therapy ${ }^{51,52}$. Therefore, CRISPR-based diagnostics reported so far largely rely on the preamplification of the target. Although some studies use $\mathrm{PCR}^{37,38}$, isothermal amplification techniques are more compatible with POC use, owing to simpler instrumentation requirements (Table 3). Some of these methods function at near-ambient temperature (in particular,
RPA, $37-42^{\circ} \mathrm{C}$ ), but others require heating devices (in particular, NASBA, $40-55^{\circ} \mathrm{C}$; LAMP, $60-65^{\circ} \mathrm{C}$; strand-displacement amplification $^{53}$ (SDA), $60^{\circ} \mathrm{C}$; helicase-dependent amplification ${ }^{54}$ (HDA), $65^{\circ} \mathrm{C}$; and exponential amplification reaction ${ }^{55}$ (EXPAR), $55^{\circ} \mathrm{C}$ ). In CRISPR-based diagnostics, the most commonly used isothermal amplification methods are RPA and LAMP. Initially, SHERLOCK ${ }^{32}$ and DETECTR ${ }^{36}$ used RPA for preamplification, which exhibited greater sensitivity than NASBA ${ }^{32}$. Although non-specific amplification represents a challenge in conventional RPA, the downstream crRNA-based target detection improves specificity. The design of RPA primers is less complex than the design of LAMP primers, but reliable procurement of the proprietary enzyme mix of a single supplier has been challenging. Furthermore, the addition of macromolecular crowding agents (such as poly(ethylene glycol)) together with low reaction temperatures reduces the mixing of the reagents and requires a mixing step for the amplification of lowly abundant targets ${ }^{56}$.

One-pot detection by using HOLMESv2 is possible by combining AacCas12b with LAMP ${ }^{39}$. During the COVID-19 pandemic, reverse transcriptase LAMP (RT-LAMP) has been widely used as an isothermal preamplification technique. With RT-LAMP, a higher operating temperature than used in RPA enables increased specificity of amplification, and the commercial availability of the necessary enzymes and reagents facilitates the planning and performing of experiments. The design of LAMP primers can be complex, but online tools (https://primerexplorer.jp and https://lamp.neb.com) can help implement this amplification technique.

\section{Quantification strategies}

In many clinical settings, an accurate quantification of the biomarker concentration is desirable, especially if changes indicate treatment efficacy such as during the monitoring of viral load under antiviral therapy ${ }^{57}$. For PCR, quantification can be absolute or relative. In absolute quantification, serial dilutions of an external standard with known concentrations are performed to obtain a standard curve, which is used to determine the target concentration in the unknown sample ${ }^{58}$. In relative quantification, which is often used to determine differentially expressed genes, the target concentration is expressed in relation to a reference ${ }^{59}$.

In CRISPR-based diagnostics, absolute quantification through comparison with a standard curve can be achieved within the picomolar-to-micromolar range $\left(10^{-12} \mathrm{M}-10^{-6} \mathrm{M}\right)$, where CRISPR-based collateral-cleavage activity correlates with target concentration $^{32}$. However, clinically useful assays generally require a lower LOD and thus preamplification. In a two-pot reaction, saturation during preamplification can prevent a quantitative readout ${ }^{32}$. 


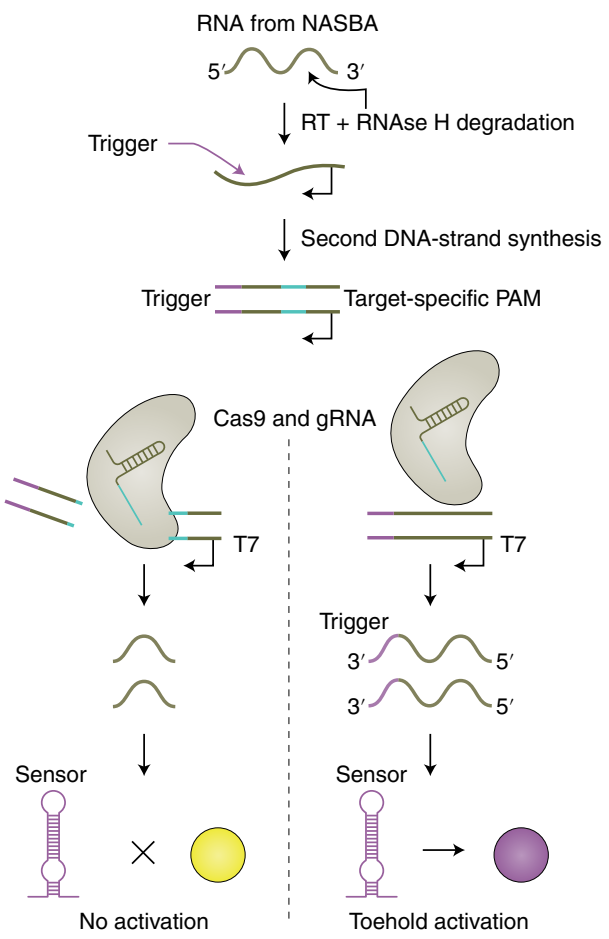

NASBACC

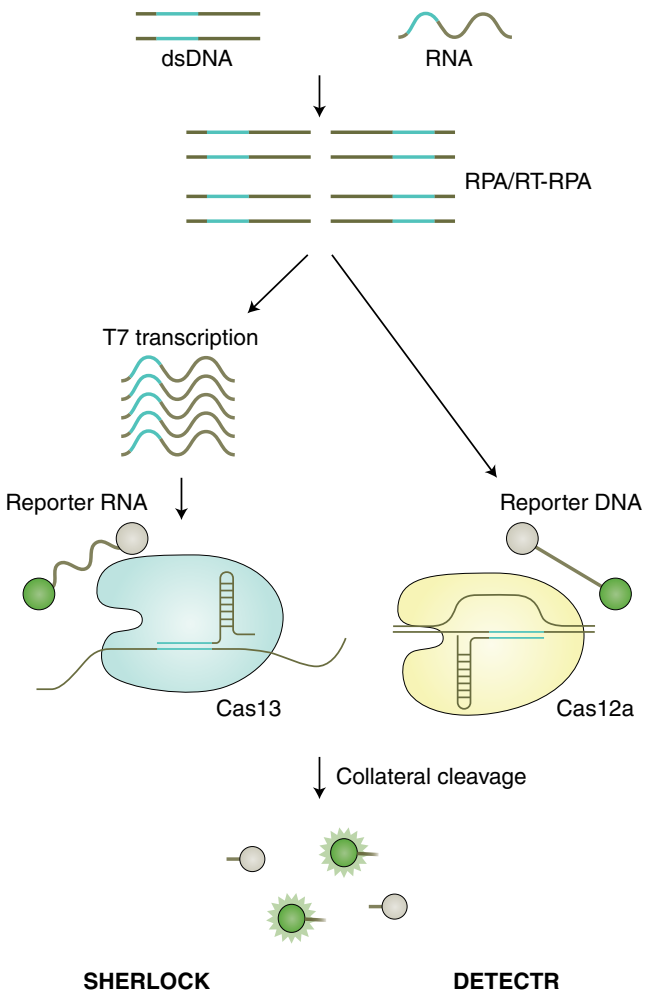

Fig. 1 | Two strategies for CRISPR-based diagnostics. Left: RNA targets are amplified through NASBA, which starts with reverse transcription (RT) to complementary DNA using a sequence-specific primer that appends a trigger sequence (magenta) for the toehold sensor. The RNA from the RNA/DNA hybrid is destroyed by RNase $\mathrm{H}$, enabling a primer that contains a T7 promoter to bind and create a complementary second DNA strand. T7 transcription of the dsDNA template creates the target RNA sequence, which can be used as starting material for a new NASBA cycle or detected by the toehold sensor. If a PAM sequence (blue) is present in the dsDNA amplicon, Cas9-mediated cleavage leads to a truncated template for T7 transcription, which generates a shorter target RNA that cannot activate the toehold sensor. In the absence of the PAM sequence, a full-length target RNA containing the trigger is transcribed, which activates the toehold sensor and produces a visible change in colour. Right: DNA or RNA are amplified by RPA or RT-RPA, respectively. For RNA-targeting CRISPR enzymes (including Cas13a), the amplified RPA product is T7-transcribed into RNA. Binding of the crRNA to the complementary target sequence activates the Cas enzyme and triggers collateral cleavage of quenched fluorescent reporters. Thereby, Cas $13 a$ (used in SHERLOCK) or Cas12a (used in DETECTR) indicate the presence of RNA or DNA target sequences, respectively.

For one-pot reactions, where preamplification and CRISPR-based detection are combined, collateral cleavage happens simultaneously with target amplification $22,34,39,60-63$. This enables a real-time readout that is amenable for quantification when a continuous measurement of the signal is incorporated. However, it requires more sophisticated laboratory equipment (similar to qPCR), which is usually not available for POC tests. To facilitate quantification in two-pot reactions, lower RPA-primer concentrations can be used to prevent saturation of the preamplification reaction ${ }^{22}$. In the SHERLOCKv2 protocol, a $240 \mathrm{nM}$ primer concentration allows for the correlation of RNA input concentration and output signal within the attomolar-to-picomolar range $\left(10^{-18} \mathrm{M}-10^{-12} \mathrm{M}\right)$. Additional options for quantification include the possibility of serial dilutions of the unknown sample compared with a known standard. The implementation of these different quantification solutions with POC-amenable devices will probably be a major focus of research in CRISPR-based diagnostic technologies. Also, controlling for the quality of nucleic acid preparations and for any inhibitory components introduced during sample collection or processing (such as heparin, excess salts, urea, haem, ionic detergents, alcohols and phenols) remains important for CRISPR-based diagnostics to reach the clinical utility of current diagnostic PCR protocols ${ }^{64}$.

\section{Strategies for the detection of multiple targets}

Comprehensive and simultaneous testing of multiple targets (multiplexing) is desirable in many diagnostic settings, as it can provide more information at higher throughput and at a lower cost per target ${ }^{5}$. Although sequencing technologies enable multiplexing through the addition of unique identifiers that are deconvoluted during data analysis ${ }^{65}$, PCR-based nucleic acid detection can be multiplexed through the simultaneous amplification of different targets in one reaction combined with the specific detection of each amplicon $^{66}$, or through the parallel readout of multiple separate reactions.

Initial efforts in the design of multiplexed CRISPR-based diagnostics took advantage of the specificity of different Cas enzymes with regards to their collateral-cleavage activity towards different nucleic acid reporter sequences. This allowed multiple nucleic acid targets to be detected by Cas enzymes within one assay ${ }^{22}$. In particular, SHERLOCKv2 enabled the qualitative detection of up to four different targets in one multiplexed reaction by including four reporter-molecule designs that were specific to the orthogonal collateral-cleavage base preferences of PsmCas13b, LwaCas13a, CcaCas13b (Capnocytophaga canimorsus Cc5) and AsCas12a (from Acidaminococcus sp.). Multiplexed Cas13 detection could be combined with multiplexed preamplification (using RPA), allowing for the simultaneous detection of two different targets (Fig. 2, left). Additional multiplexing beyond these levels in a single reaction was limited by the preamplification chemistries ${ }^{22}$.

The CARMEN (for combinatorial arrayed reactions for multiplexed evaluation of nucleic acids) CRISPR-based multiplexed assay enables the scalable and parallel detection of more than 
Table 3 | Isothermal amplification methods

\begin{tabular}{|c|c|c|c|c|}
\hline Method & RPA & LAMP & SDA & NASBA \\
\hline Temperature $\left({ }^{\circ} \mathrm{C}\right)$ & $37-42$ & $60-65$ & $37-60$ & 65 (priming); 41 \\
\hline Time (min) & $20-60$ & $20-60$ & $30-60$ & $60-120$ \\
\hline Involved proteins & $\begin{array}{l}\text { Recombinase, single-strand binding protein, } \\
\text { strand-displacing DNA polymerase }\end{array}$ & $\begin{array}{l}\text { Strand-displacing } \\
\text { DNA polymerase }\end{array}$ & $\begin{array}{l}\text { Strand-displacing DNA } \\
\text { polymerase, nicking } \\
\text { endonuclease }\end{array}$ & $\begin{array}{l}\text { Reverse transcriptase, RNase } \\
\mathrm{H}, \text { T7 RNA polymerase }\end{array}$ \\
\hline Primers & 2 & $6-8$ & 4 & 2 \\
\hline
\end{tabular}

aRNA can be detected by introducing a reverse-transcriptase enzyme into the reaction. ${ }^{\mathrm{b}} \mathrm{Although}$ NASBA is primarily used for the amplification of RNA, modifications of the protocol have enabled DNA amplification.

4,500 targets ${ }^{67}$. The method builds on the reaction chemistry of SHERLOCK, yet uses a miniaturized reaction volume (Fig. 2, right). In CARMEN, amplified nucleic acid target samples and LwaCas 13 detection mixes are each combined with a distinct solution-based colour code. The colour-coded solutions are emulsified in fluorous oil, creating nanolitre droplet inputs. The droplets from all of the samples and detection mixes are pooled and subsequently loaded onto a microfabricated chip to create all possible pairwise combinations of sample and detection droplets (one pair per well). Droplet pairs are then merged by applying an external electric field. An initial recording of the dyes of two droplets in a particular microwell indicates which sample and detection droplet are present, and fluorescence generated through Cas13a-triggered reporter cleavage on target detection indicates a positive reaction. This miniaturization coupled with the high sensitivity and specificity of Cas13a enabled the detection of 169 human-associated viruses, the subtyping of coronaviruses and influenza A strains, and the identification of HIV mutations that are drug resistant. Compared with traditional DNA microarrays, CARMEN does not require the time-consuming spotting of probes and offers higher scalability than PCR panels. Although it requires a preamplification step, microscopy analysis and skilled lab personnel, CARMEN allows for fast, high-throughput and low-cost pathogen detection, which is critically needed in clinical routines and for the surveillance of emerging public-health threats. Owing to the miniaturized reactions for detection, CARMEN considerably reduces reagent costs and sample consumption. However, its current dependence on advanced microscopy limits the use of the technology to resource-rich laboratory settings.

\section{Combination of CRISPR-Cas systems with sequencing}

Because sequencing-based diagnostic technologies use the alignment of reads to bioinformatic databases for pathogen identification, they tolerate variability in the genome of the target organism and do not necessarily require prior knowledge of the target sequence. These features are particularly useful when the pathogen is unknown, or when its genome evolves quickly. However, to detect low-abundance pathogens, the enrichment of regions of interest before sequencing becomes necessary. PCR-based amplification of sequencing regions has several limitations, including the removal of epigenetic marks, amplification bias and challenges associated with the amplification of GC-rich regions or of very large regions. Larger amplicons are of particular interest for long-read sequencing techniques, such as nanopore sequencing, because they allow for the characterization of long-range structural variations.

To overcome these limitations, several protocols use Cas nucleases to enrich regions of interest before sequencing. For example, nanopore Cas9-targeted sequencing (nCATS) uses Cas9 to cleave chromosomal DNA for the ligation of adapters for nanopore sequencing (Fig. 3, left), enabling the targeted sequencing of long fragments at high depth while maintaining epigenetic marks ${ }^{68}$. This technology allowed the simultaneous detection of CpG methylation, structural variations and haplotype-resolved single-nucleotide variants using $3 \mu \mathrm{g}$ of genomic DNA, and it achieved $675 \times$ coverage with a MinION flow cell and $34 \times$ coverage with the Flongle flow cell (both cells are commercialized by Oxford Nanopore Technologies). In a similar effort to circumvent the need for PCR-based enrichment, STRique (for short tandem repeat identification, quantification and evaluation) was developed ${ }^{69}$ for the identification of short tandem repeats (STRs) with nanopore sequencing (Fig. 3, left). In addition to Cas9, Cas12a was used to induce DNA cuts next to a repeat-containing region, followed by the ligation of adapters for nanopore sequencing. Using an algorithm that identified the positions of the STR-flanking regions and the number of STRs between them, the method enabled the detection of STR expansion alongside $\mathrm{CpG}$ methylation status in cell lines derived from patients with fragile X syndrome and with amyotrophic lateral sclerosis.

CRISPR-Cas systems have also been combined with targeted next-generation sequencing to increase the sequencing yield. For example, DASH (for depletion of abundant sequences by hybridization) was developed ${ }^{70}$ to enrich pathogen sequences and to remove unwanted species. In this method, a library of crRNAs directs Cas9 to unwanted target sequences for cleavage, and non-targeted regions retain the intact adapters required for amplification and sequencing (Fig. 3, right). Another method, FLASH (for finding low-abundance sequences by hybridization), includes the blocking of gDNA by phosphatase and digestion through Cas9 complexed to a set of crRNAs targeting genes of interest, followed by the ligation of adapters, and by amplification and sequencing ${ }^{71}$. This technique detected, in respiratory fluids and dried blood spots, subattomolar levels of drug-resistant Gram-positive bacteria and the malaria parasite Plasmodium falciparum.

\section{Assay optimization for applications at the POC and in the field}

Readouts. Some strategies for measuring and visualizing the activation of Cas enzymes on target recognition have employed fast and low-cost readouts amenable for POC or field applications. The usage of reporter molecules with different properties enables a wide variety of readouts, including fluorescence, lateral-flow immunochromatography and electrochemistry. For fluorescence-based assays, most CRISPR-based diagnostic methods take advantage of RNA or DNA reporter molecules carrying a fluorophore and a quencher. Collateral-cleavage activity on target detection causes the spatial separation of the fluorophore and the quencher, and the resulting fluorescence can be read on conventional plate readers (Fig. 4, top left), on low-cost and portable devices ${ }^{72}$ and even with the naked eye under blue light ${ }^{60}$ (Fig. 4, top left). Additional strategies enable colorimetric visualization by using gold nanoparticles, which are 
Pooled multiplexing
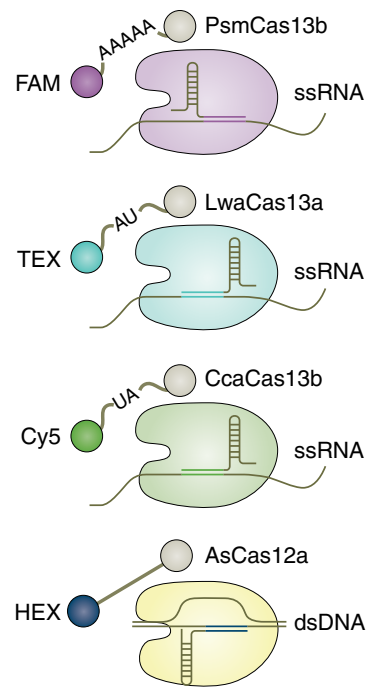

$\downarrow$ in pooled reaction
Arrayed multiplexing

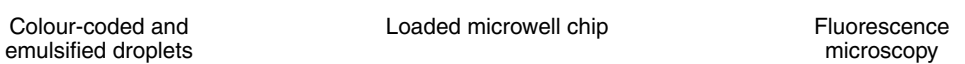

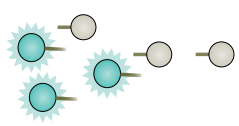
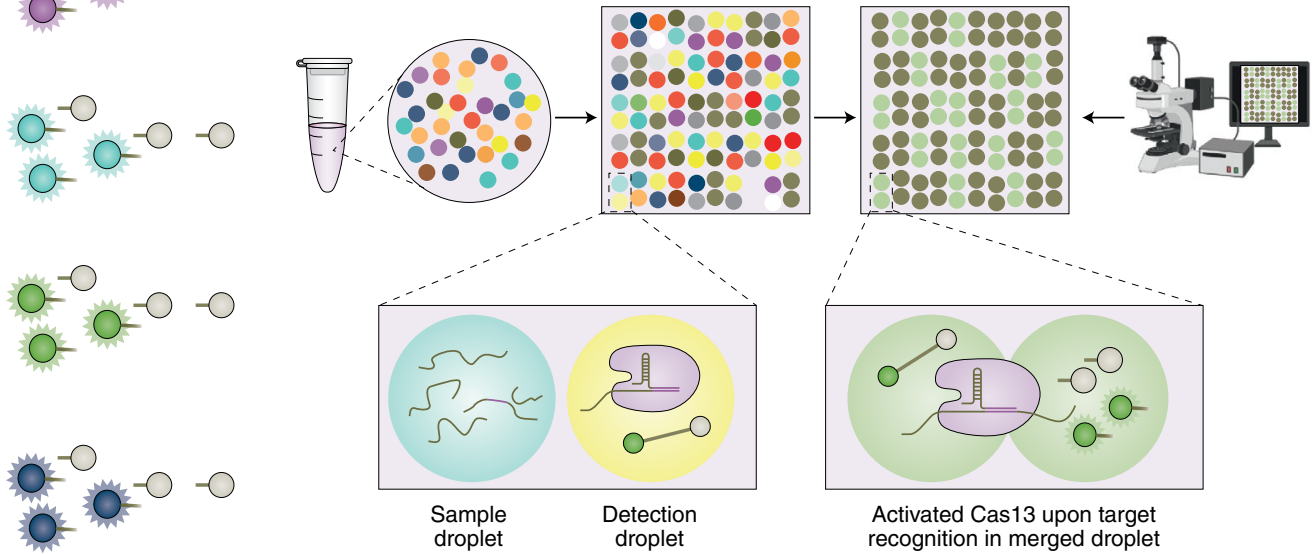

Activated Cas13 upon target recognition in merged droplet

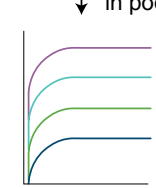

Electric field

Fig. 2 | Multiplexed CRISPR-based target detection. Left: pooled multiplexing of up to four targets, as implemented in SHERLOCKv2. Orthogonal CRISPR enzymes (PsmCas13b, LwaCas13a, CcaCas13b, AsCas12a), each cleaving preferentially different reporter molecules bearing different fluorophores (FAM, TEX615 (TEX), Cy5, hexachlorofluorescein (HEX)) with distinct absorbance and emission wavelengths. Right: in CARMEN, a distinct colour code is added to each PCR-amplified sample or Cas13-based detection mix. The Cas13-based detection mix contains Cas13a, a sequence-specific crRNA and a cleavage reporter. The colour-coded solutions are emulsified in fluorous oil, which creates nanolitre droplets. Droplets from all samples and detection mixes are pooled together and loaded into a microwell-array chip in one step to create all possible pairwise combinations. The droplet pair in each well is identified using fluorescence microscopy before they are merged by exposure to an electric field. Fluorescence microscopy is then used to monitor each Cas13-based detection reaction.

linked through ssRNA or ssDNA oligonucleotides. Cas12-mediated or Cas13-mediated trans cleavage of the linker resulted in the dispersal of the gold nanoparticles on target recognition, and produced a vibrant red colour in solution ${ }^{73}$. Alternatively, Cas-mediated cleavage of biotinylated ssDNA substrates resulted in a decrease in the magnetic pull-down of DNA gold nanoparticles ${ }^{74}$. A turbidity-based assay based on the liquid-liquid phase separation of nucleic acids and positively charged polyelectrolytes can also provide visual readouts $^{75}$. In this case, collateral cleavage leads to the degradation of nucleic acid polymers on target recognition. This can be visualized after complementation with polycations, where the solution either stays clear when cleavage has occurred or becomes turbid in the absence of target-triggered cleavage.

Lateral-flow assays (Fig. 4, top right) offer a simple visualization of the collateral cleavage activity inherent to many CRISPR-based diagnostics. A commercially available system (Millenia 1T) first reported in ref. ${ }^{22}$ has been widely adopted. In this technique, reporter molecules carrying biotin and fluorescein (fluorescein amidite (FAM)) bind to anti-fluorescein isothiocyanate (FITC) antibodies coupled to gold nanoparticles in the sample pad area of the strip. These complexes subsequently travel to a streptavidin-capture line, where they bind through the biotin of the reporter molecule. In the absence of the target, uncut reporter molecules are retained there, and this is visualized as one band on the strip. In the presence of the target, Cas-enzyme-mediated collateral cleavage of the reporter separates the biotin from the FAM molecules bound to the anti-FITC gold nanoparticles, allowing them to travel farther on the strip and generating a second visible band at the antibody-capture line, which bears species-specific secondary antibodies. An alter- native to collateral cleavage-based target detection combines Cas9-based amplicon detection with a lateral-flow readout using a crRNA-anchoring-based hybridization assay ${ }^{76}$.

CRISPR-based diagnostics with electronic readouts are also possible. One method, named CRISPR-Chip, involved deactivated Cas9 complexed with a target-specific crRNA and immobilized on a graphene-based field-effect transistor ${ }^{45}$. On target binding, alterations to graphene's conductivity caused the electrical properties of the transistor to change, which can be measured as a change in current. Notably, this technology did not require labelled reporter molecules and could sense deletions in the dystrophin gene in unamplified gDNA samples from patients with Duchenne muscular dystrophy. The sensitivity of the system reached the femtomolar range $(\sim 1.7 \mathrm{fM})$. Another strategy for obtaining electronic readouts, named E-CRISPR, involved an ssDNA-cleavage reporter with a methylene blue electrochemical tag and a thiol moiety for immobilization on a gold electrode ${ }^{77}$. In this method, Cas12a-mediated collateral cleavage upon target detection led to a decrease in the electrochemical current through the release of methylene blue, whereas the absence of target resulted in a high electrochemical current, owing to an intact methylene blue-carrying reporter. This assay detected human papillomavirus and parvovirus B19 DNA with a LOD of $\sim 50 \mathrm{pM}$, and can be adapted for protein sensing. Another method used an electrochemical biosensor to detect Cas13a-mediated collateral cleavage of RNA reporters carrying FAM and biotin ${ }^{46}$ (Fig. 4, bottom left). In this approach, the binding of glucose-oxidase-coupled anti-FAM antibodies to reporter molecules immobilized on the sensor catalysed the oxidation of glucose. The produced $\mathrm{H}_{2} \mathrm{O}_{2}$ was then amperometrically detected by using 

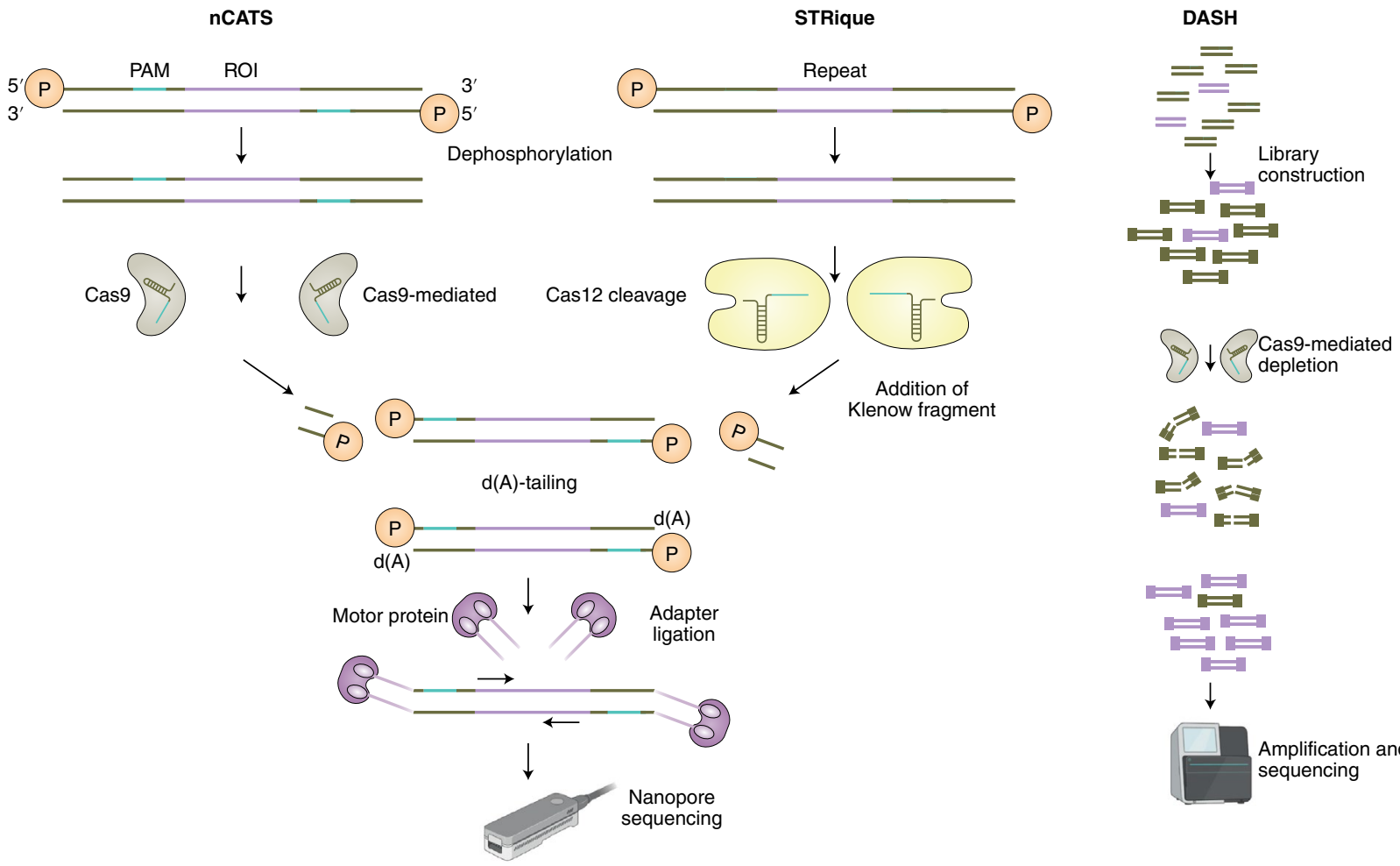

Fig. 3 | CRISPR-Cas-mediated enrichment strategies for sequencing-based diagnostics. Left: nCATS is a Cas9-based enrichment technology, which first dephosphorylates DNA ends and then uses Cas9 to induce cuts near a region of interest (ROI) that contains a PAM sequence. A DNA polymerase I with an $\mathrm{N}$-terminal truncation that lacks exonuclease activity (Klenow fragment) enables the addition of dAMP (deoxyadenosine monophosphate (dA)) to the $3^{\prime}$ end the DNA fragment ( $(\mathrm{A})$-tailing)). Next, adapters containing a motor protein are ligated to the DNA at Cas 9 cleavage sites, enabling the DNA to pass through the nanopore. STRique uses Cas12a for the enrichment of repeat-containing regions analysed by nanopore sequencing, and employs an algorithm for the identification of the position of the repeat flanking regions and the number of repeats. Right: DASH uses Cas 9 to deplete unwanted sequences (black) before next-generation sequencing. Non-targeted sequences (magenta) retain intact adapters for amplification and sequencing.

an electrochemical cell with a platinum working electrode, a platinum counter electrode and a silver/silver chloride reference electrode. This technique allowed the detection of miRNAs at a LOD of $10 \mathrm{pM}$ within 4 hours in volumes smaller than $0.6 \mu \mathrm{l}$ (this LOD was achieved using a separate 'off-chip' cleavage; the all-in-one sensor had a LOD of $2.2 \mathrm{nM}$ ). Cas13a-based electrochemical detection of miRNAs was also shown with a portable electrochemiluminescence $\operatorname{chip}^{78}$ (with a reported limit of detection of $1 \mathrm{fM}$ ). In this technique, the miRNA-mediated activation of Cas13a led to the collateral cleavage of a trigger for the initiation of exponential isothermal amplification. The amplified products formed a complex with $[\mathrm{Ru}(\mathrm{phenan}$ throline) ${ }_{2}$ dipyridophenazine $]^{2+}$, and the oxidation of the complex at the anode of a bipolar electrode generated an electrochemiluminescent signal proportional to the concentration of the target miRNA.

DNA hydrogels have been designed to enable CRISPR-based diagnostic readouts through changes in their material property ${ }^{79,80}$. The hydrogels consist of water-filled polymers containing DNA molecules serving as an anchor or structural element within the gel (Fig. 4, bottom right). On target recognition, Cas12a triggers collateral cleavage of the DNA molecules, which changes the properties of the gel. This technology was used for sensing pathogen-derived nucleic acids in a multilayered paper-based microfluidic device $(\mu \mathrm{PAD})$ with dual visual and electronic readouts. In the absence of the target, the DNA-linker-containing hydrogel obstructed the flow of a buffer through the porous channels of the paper-based device. Target recognition led to Cas12a-mediated linker destruction, which prevented hydrogel crosslinking and caused visually observable flow through the porous $\mu \mathrm{PAD}$ channels. This technique allowed for the detection of dsDNA down to $400 \mathrm{pM}$ without preamplification. To create an electronic readout, two electrodes were placed in the bottom layer of the $\mu \mathrm{PAD}$. Because the electrical conductivity of the gel depended on its degree of crosslinking, low levels of crosslinking due to Cas12a-mediated cleavage caused an electrolyte-containing buffer to flow, whereas high levels of crosslinking in the absence of any target blocked the buffer from flowing through, thereby preventing the recording of an electric signal. Moreover, the incorporation of a wireless radiofrequency identification (RFID) module into the $\mu \mathrm{PAD}$ enabled the monitoring of target detection at different geographic locations in real time. To this end, Cas12a-mediated collateral cleavage on target detection resulted in the short-circuiting of an interdigitated electrode arrangement in the loop RFID tag, which increased the strength of the received signal with respect to that of a reference RFID tag.

Sample preparation. Sample processing is a critically important consideration for improving the usability of CRISPR-based diagnostics for field applications and POC settings. CRISPR-based diagnostics initially used synthetic targets or time-consuming or equipment-intensive nucleic acid isolation protocols. More recent efforts aim for the rapid and equipment-free isolation of DNA or RNA. Importantly, the reagents used in the sample-extraction steps must not interfere with any downstream preamplification steps or with CRISPR-based target detection. As in many cases, the final readout is based on collateral-cleavage activity, and special precautions must be taken to ensure the absence of RNases and DNases, which can cleave ssRNA or ssDNA reporters.

The original SHERLOCK protocol showed that saliva could be processed directly by using $0.2 \%$ Triton $\mathrm{X}-100$ in a lysis mixture 
Fluorescence

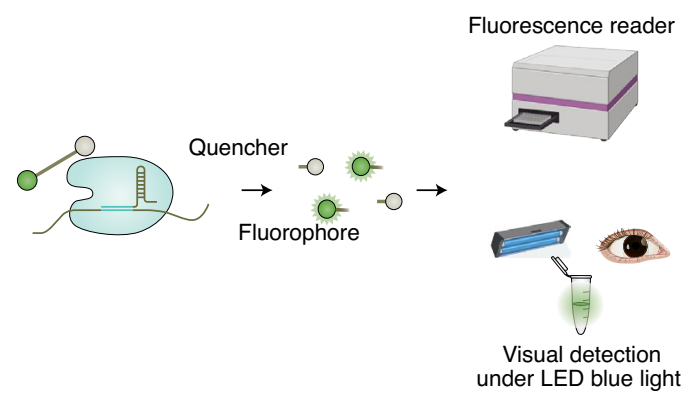

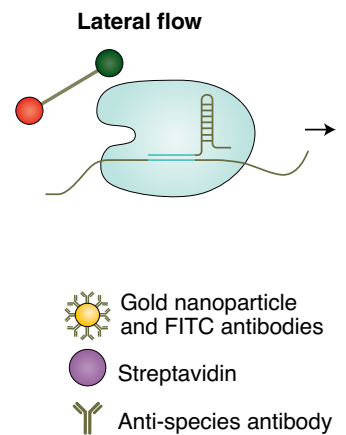
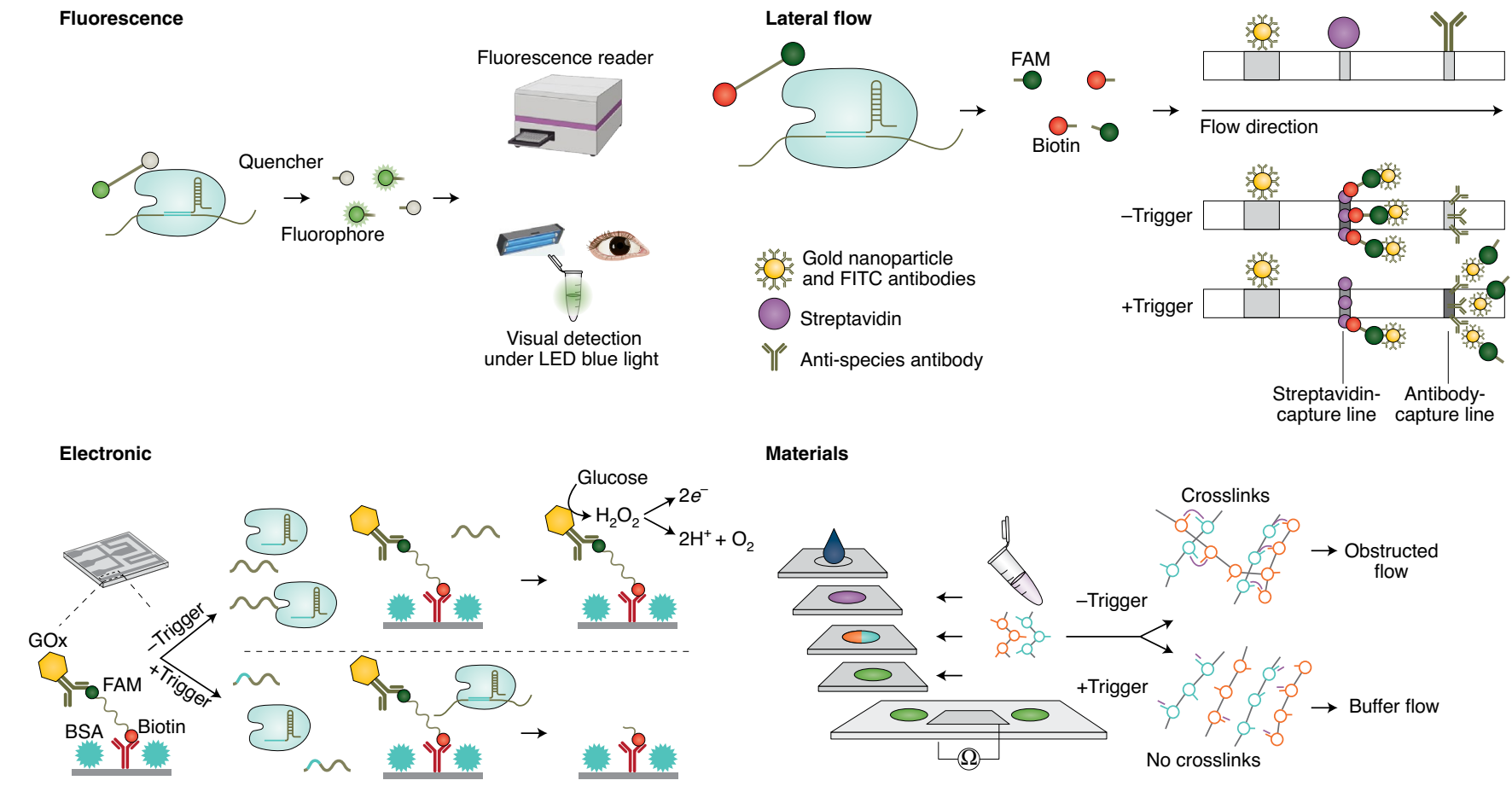

Materials

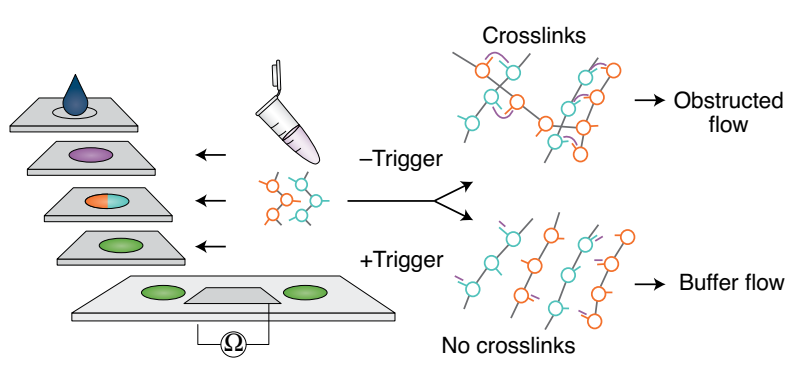

Fig. 4 | Collateral-cleavage readouts. Top left: fluorescence readouts rely on quenched fluorophores that, on target recognition, are released as a result of the collateral cleavage of the DNA or RNA oligomers joining the fluorophore and quencher. The signal can be measured by a fluorescence reader or read by eye under blue light. LED, light-emitting diode. Top right: lateral-flow-based immunochromatographic readouts rely on the high affinity of streptavidin and biotin, and use a reporter molecule consisting of a DNA or RNA oligomer conjugated to FAM and biotin. The product of the CRISPR reaction is deposited on the sample-application area of a test strip, at which point FAM binds to gold-nanoparticle-labelled FITC-specific antibodies. When intact, the reporter oligomer remains bound to the streptavidin line through biotin, creating one colour band on the test strip. When the reporter oligomer is cleaved, FAM and the bound gold-labelled FITC-specific antibodies flow farther on the strip and bind to secondary anti-species antibodies, which leads to the formation of a second colour band. Bottom left: electrochemical readout relying on the amperometric detection of $\mathrm{H}_{2} \mathrm{O}_{2}$ in an electrochemical cell ${ }^{46}$. Anti-fluorescein antibodies (green) coupled with glucose oxidase (GOx) bind to FAM-carrying RNA reporter molecules, which are immobilized through biotin on a surface. The concentration of GOx is reduced in the presence of an RNA trigger, owing to the activated crRNA/Cas13a complex cleaving the RNA reporter molecule on target recognition, which frees the GOx-carrying anti-fluorescein antibodies from the surface. Bottom right: in the presence of a dsDNA trigger, Cas12a collaterally cleaves ssDNA linkers within a hydrogel. This prevents crosslinking within the hydrogel, thereby modulating the permeability of a paper-based microfluidic device and allowing for buffer flow, which can be measured electrically ${ }^{79,80}$.

with phosphate buffered saline, and by heating at $95^{\circ} \mathrm{C}$ for $5 \mathrm{~min}$. This crude saliva mixture was used in a SHERLOCK reaction for genotyping. To expand to additional sample types, the protocol HUDSON (for heating unextracted diagnostic samples to obliterate nucleases) enabled the inactivation of nucleases and viruses through heat and chemical reduction through the addition of tris(2-carboxyethyl)phosphine hydrochloride at a $100 \mathrm{mM}$ final concentration and ethylenediaminetetraacetic acid (EDTA) at a $1 \mathrm{mM}$ final concentration ${ }^{81}$. Different heating steps (ranging from $5 \mathrm{~min}$ to $20 \mathrm{~min}$ and from $37^{\circ} \mathrm{C}$ to $95^{\circ} \mathrm{C}$ ) were optimized for different targets and sample types. HUDSON enabled the detection of RPA-preamplified targets through CRISPR-Cas13a, including the sensing of ZIKV and dengue virus in blood, plasma, serum, saliva and urine. Since the HUDSON protocol was not sufficient to inactivate nucleases for the detection of Plasmodium species (as observed by the fraction of false-positive samples), a preparation buffer containing stronger chelating agents has been developed $(20 \% \mathrm{w} / \mathrm{v}$ Chelex100 TE-buffer containing dithiothreitol (DTT) at $50 \mathrm{mM})^{61}$.

A commercially available fast DNA-extraction solution (QuickExtract, Lucigen) enables fast RNA preparation for the detection of SARS-CoV-2 RNA through RT-qPCR ${ }^{82}$. Here nasopharyngeal swabs are mixed with the extraction solution, incubated at $95^{\circ} \mathrm{C}$ for $5 \mathrm{~min}$ and directly used in the RT-qPCR reaction after cool down. This isolation protocol is compatible with downstream RT-LAMP preamplification and detection through Cas12b from Alicyclobacillus acidiphilus (AapCas12b) ${ }^{63}$. QuickExtract also enabled the lysis of samples at $22^{\circ} \mathrm{C}$ or $60^{\circ} \mathrm{C}$ for $10 \mathrm{~min}$, and when proteinase $\mathrm{K}$ was blocked in the downstream reactions by an inhibitor.

Large-scale testing and costs. One advantage of CRISPR-based diagnostic assays is their independence from complex and costly laboratory equipment and from common reagents such as PCR master mixes. Because Cas enzymes and crRNAs can be produced fast and at scale, CRISPR-based assays are less dependent on supply-chain issues. However, although the costs of buffers, primers and the oligos used as templates for crRNA synthesis are negligible, CRISPR-based assays include many more enzymes than PCR, especially when an isothermal preamplification step is included. The cost for an individual CRISPR-based diagnostics reaction with preamplification is approximately US\$0.61 per reaction in an experimental setting $^{32}$. Of these costs, RPA reagents made up the largest fraction. However, the first commercially available CRISPR-based diagnostic assay for SARS-CoV-2 including RT-LAMP as preamplification is currently available at US\$30.15 per reaction (https://www.idtdna. $\mathrm{com} /$ pages/landing/coronavirus-research-reagents/sherlock-kits). It is unclear how prices will develop after the approval of more tests, and how patents that cover methods for the sensing of nucleic acids through CRISPR-Cas proteins will affect the dissemination of the technology. 
Most CRISPR-based diagnostic assays use liquid reagents, which afford experimental flexibility. However, the storage of solutions of the crRNA, cleavage reporter and Cas protein typically requires freezers that maintain ultralow temperatures. Lyophilization of these components and of the preamplification reagents eliminates the dependence on cold chains, and by using higher input volumes of sample the assay sensitivities can be similar ${ }^{31}$ or even higher ${ }^{61}$ than with the use of non-lyophilized reactions. Systematic stability testing of the components for CRISPR-based diagnostics is needed, yet studies in clinical cohorts ${ }^{83}$ and the assessment of daily experimental variation $^{84}$ suggest that the results of the assays are highly reproducible.

\section{Biomedical applications}

CRISPR-based diagnostics have been used for a wide range of biomedical applications, and in particular for the sensing of nucleic-acid-based biomarkers of infectious and non-infectious diseases and for the detection of mutations and deletions indicative of genetic diseases. Moreover, the technology has been adapted for the sensing of proteins and small molecules.

Infectious diseases. The main focus of CRISPR-based diagnostics has been the detection of pathogens; in particular, the DNA or RNA of viruses, bacteria and parasites. RNA viruses sensed with CRISPR-based methods include parvovirus B19 (ref. ${ }^{77}$ ), members of the family Flaviviridae (dengue ${ }^{22,25,81}, \mathrm{Zika}^{25,32}$ and Japanese encephalitis virus ${ }^{37}$ ), Ebola ${ }^{85}$ and Coronaviridae (which have naturally become a major focus of interest during the COVID-19 pandemic).

The SHERLOCK-based detection of SARS-CoV-2 initially consisted of a two-step assay comprising RPA-based preamplification, followed by $\mathrm{T} 7$ transcription and Cas13a-mediated target recognition $^{86}$. This assay has been tested in a qPCR-validated clinical cohort containing 154 samples analysed by lateral-flow and fluorescence readouts, and with 380 PCR-negative samples analysed by fluorescence only ${ }^{83}$. The assay reached a LOD of 42 RNA copies per reaction, and sensitivities of $96 \%$ and $88 \%$ when using fluorescence and lateral-flow readouts, respectively. Both readouts were $100 \%$ specific. In May 2020, a modified SHERLOCK-based two-step assay using RT-LAMP instead of RT-RPA received emergency use authorization from the United States Food and Drug Administration for the CRISPR-based detection of SARS-CoV-2. Testing with this assay is intended for the qualitative detection of SARS-CoV-2 in specimens of the upper respiratory track, and is limited to laboratories authorized by CLIA (for clinical laboratory improvement amendments) regulations. A different Cas13a-based assay, SHINE (for SHERLOCK and HUDSON integration to navigate epidemics) ${ }^{62}$, combined the preamplification reaction and CRISPR-based detection into a single-step reaction that uses HUDSON to accelerate SARS-CoV-2 viral extraction in nasal swabs and saliva samples. The addition of RNase $\mathrm{H}$, the optimization of buffers, concentrations of magnesium and of primers, and the usage of a polyU reporter together with a SuperScript-IV reverse transcriptase, reduced the LOD to a range similar to that of the two-step assay (10 copies per $\mu \mathrm{l}$ with the fluorescent readout, and 100 copies per $\mu \mathrm{l}$ with the lateral-flow readout). The test was validated on 50 nasopharyngeal patient samples, and achieved $90 \%$ sensitivity and $100 \%$ specificity against RT-PCR. In a different Cas13-based approach, the integration of multiple crRNAs, the usage of a Cas13a homologue from Leptotrichia buccalis ( $\mathrm{Lbu}$ ) and the measurement of fluorescence over time enabled the detection of SARS-Cov-2 RNA extracted from nasal swabs down to $1.27 \times 10^{8}$ copies per $\mathrm{ml}\left(1.65 \times 10^{3} \mathrm{cop}\right.$ ies per $\mu l$ in the Cas 13 reaction). Importantly, this was achieved without the need for preamplification, and the assay was compatible with readout using a portable fluorescence-detection device ${ }^{44}$.

By using Cas12a, the two-step assay SARS-CoV-2 DETECTR was optimized to detect the $\mathrm{N}$ (nucleoprotein) and $\mathrm{E}$ (envelope small membrane protein) genes of the SARS-CoV-2 genome, as well as a human control gene (for the ribonuclease $\mathrm{P}$ protein), in less than $40 \mathrm{~min}$ from respiratory swab RNA extracts preamplified by RT-LAMP ${ }^{87}$. SARS-CoV-2 DETECTR returned results with a $95 \%$ positive predictive agreement and a $100 \%$ negative predictive agreement relative to the RT-qPCR assay from the United States Centers for Disease Control and Prevention. Cas12a was also used in the SARS-CoV-2 AIOD-CRISPR (for all-in-one dual CRISPR-Cas12a) assay ${ }^{60}$, which uses Cas12a together with a pair of non-PAM-dependent crRNAs, each sensing separate regions of the target nucleic acid amplicon. These Cas12a-crRNA complexes were introduced into a one-pot assay containing the reagents for RPA-based preamplification and the cleavage reporter. This approach enabled the detection of 5 copies of synthetic SARS-CoV-2 RNA per reaction, and was validated in 28 clinical swab samples. STOPCovid (for SHERLOCK testing in one pot) ${ }^{63}$, another Cas12-based one-pot assay, integrated RT-LAMP-based preamplification with CRISPR-mediated detection in a one-step workflow. Of the various Cas proteins explored, Cas12b from Alicyclobacillus acidiphilus (AapCas12b) together with the scaffold single guide RNA from AacCas12b, allowed the test to run at the temperature range required for LAMP. STOPCovid has a sensitivity comparable to that of RT-qPCR, with a LOD of 100 copies of viral genome per reaction.

Besides RNA viruses, DNA viruses such as Herpesviridae (including cytomegalovirus $(\mathrm{CMV})^{84}$ and Epstein-Barr virus ${ }^{88}$ ), Polyomaviridae (BK virus (BKV) $)^{84}$ and Papillomaviridae (human papillomavirus) ${ }^{36}$ have also been detected with CRISPR-based diagnostics. In particular, a SHERLOCK-based assay was used to detect $\mathrm{CMV}$ and $\mathrm{BKV}$ in a clinical setting ${ }^{84}$. The assay was validated with $\mathrm{qPCR}$, and detected BKV from 67 HUDSON-isolated plasma and urine samples with $100 \%$ sensitivity and $100 \%$ specificity. HUDSON-based processing of CMV-containing plasma samples resulted in $80 \%$ sensitivity and $100 \%$ specificity, whereas DNA purification through silica-based membranes was necessary to reach $100 \%$ sensitivity and $100 \%$ specificity. To objectively assess the results of the lateral-flow assay, a smartphone-based software application was developed to quantify band intensities. The background signal of the lateral-flow assay was independent of daily experimental variation, incubation time and temperature, which facilitated the reproducibility of the qualitative readout.

Bacteria detected with CRISPR include Mycobacterium tuberculosis $^{32,89}$, Staphylococcus aureus ${ }^{22,32,90}$, Listeria monocytogenes ${ }^{91}$, Pseudomonas aeruginosa ${ }^{32}$ and Salmonella enteritidis ${ }^{92}$. CRISPR has also been adapted to sense the parasites of the Plasmodium group responsible for malaria. This includes the sensing of different $P$. falciparum strains ${ }^{71}$ and the development of a pan-plasmodium assay ${ }^{93}$ that detected all Plasmodium species known to cause malaria in humans as well as the species-specific detection of $P$. vivax and $P$. falciparum. A different CRISPR-based assay based on an RPACas12a one-pot reaction using lyophilized reagents downstream of a simplified sample preparation was developed for the detection of $P$. falciparum, $P$. vivax, $P$. ovale and $P$. malariae ${ }^{61}$. The addition of a reverse transcriptase to transcribe target RNA into DNA increased the assay's sensitivity. The protocol achieved a LOD between 0.36 and 2.4 parasites per $\mu \mathrm{l}$, depending on the Plasmodium species.

Non-infectious diseases. CRISPR-based diagnostics have also been developed for the detection of RNA species relevant to non-infectious diseases. For example, CRISPR-based sensing of human CXCL9 mRNA was used to detect acute cellular kidney-transplant rejection, as defined by renal biopsy, with a sensitivity of $93 \%$ and a specificity of $76 \%$, in total RNA isolated from 31 urinary cell pellets ${ }^{84}$. CRISPR-based diagnostics have also been used to sense miRNAs, as exemplified by the CRISPR/LwaCas13a-based electrochemical detection of miR-19b in serum samples of patients with medulloblastoma ${ }^{46}$ without preamplification, and the testing of RNA isolated 
from breast cancer cell lines for miR-17 with CRISPR/LbuCas $13 \mathrm{a}^{94}$ and for miR-21 with LbCas12a, after rolling-circle transcription ${ }^{95}$.

SNPs and deletions. A major strength of CRISPR-based diagnostics is the single-nucleotide specificity of Cas enzymes, which enables the detection of point mutations and small deletions. Single-nucleotide specificity has made possible the CRISPR-based detection of markers of antimicrobial resistance, of deletions and mutations in the epidermal-growth-factor-receptor gene ${ }^{22}$, of mutations conferring Duchenne muscular dystrophy ${ }^{45}$ and of SNPs in the E3 ubiquitin ligase gene conferring eye colour ${ }^{41}$. The single-nucleotide specificity of the CRISPR-Cas system has also been leveraged for the sensing of miRNAs, which are challenging to detect because of their short size and because they can differ by only a single base. CRISPR-based approaches may also be suitable for the detection of circulating SARS-CoV-2 variants that might differ in pathogenicity and transmissibility ${ }^{96}$.

Targets other than nucleic acids. CRISPR-based diagnostic methods have been adapted for the detection of proteins and small molecules. In these cases, the CRISPR system is primarily used as a reporter or amplifier, and the actual sensing of the target molecule is mediated through proteins or aptamers that undergo a conformational change on target recognition. One strategy for sensing small molecules combined CRISPR-Cas12a with bacterial allosteric transcription factors ${ }^{97}$ (aTFs). Referred to as CaT-SMelor (for CRISPRCas12a-mediated and aTF-mediated small molecule detector), the method was applied to sense uric acid and $p$-hydroxybenzoic acid at nanomolar concentrations, and thereby enabled the detection of uric acid in human blood samples. In this method, the incubation of immobilized aTF-dsDNA complexes containing the Cas12a target sequence with the aTF target small molecule led to a conformational change in the aTF that freed the dsDNA from the complex. After removal of the immobilized aTF by centrifugation, free dsDNA was detected using CRISPR-Cas12a and measured through the collateral cleavage of a quenched fluorescent reporter. SPRINT (for SHERLOCK-based profiling of in vitro transcription) ${ }^{98}$ used aTFs and riboswitches that are triggered by specific effector molecules to regulate transcription of RNA targets. Transcribed RNA is then detected by a crRNA and Cas13a. This enabled the sensing of a wide range of effector molecules (including fluoride, adenine, guanine, $S$-adenosylmethionine, flavin mononucleotide, serotonin, zinc and anhydrotetracycline).

A different approach to sensing small molecules involved an aptamer that contained an ssDNA target of Cas12a, which served as a sensor for adenosine triphosphate ${ }^{99}$ (ATP; reported LOD of the assay, $400 \mathrm{nM}$ of ATP). In this approach, the binding of ATP to the aptamer hindered target recognition and thereby reduced the Cas12a-triggered collateral cleavage of an ssDNA reporter. Alternatively, ATP or $\mathrm{Na}^{+}$ions have been sensed through aptamers or DNAzymes (also referred to as 'functional DNA') that release Cas12a targets on binding to their non-nucleic acid targets, resulting in the activation of Cas12a as detected by the trans cleavage of fluorescent reporter molecules ${ }^{100}$.

Similarly, an aptamer-based detection method has been developed for the CRISPR-Cas12a-based sensing of the transforming growth factor $\beta 1$ (TGF- $\beta 1$ ) protein ${ }^{77}$. In this method, TGF- $\beta 1$ binds to a specific ssDNA aptamer that contains a Cas12a target site. In the absence of TGF- $\beta 1$, all free and unbound aptamer molecules are recognized by Cas12a, whose collateral-cleavage activity of ssDNA reporters that carry methylene blue results in a decrease of the electrochemical signal, which is sensed by E-CRISPR. The aptamer-based E-CRISPR assay allowed for the detection of TGF- $\beta 1$ in clinical samples with a LOD of $0.2 \mathrm{nM}$. And CLISA (for CRISPR-Cas13a-based signal amplification linked immunosorbent assay) was developed to sense human interleukin-6 at $2.29 \mathrm{fM}$ and human vascular endothelial growth factor at $0.81 \mathrm{fM}$ (ref. ${ }^{101}$ ). This was achieved by replacing the signal-amplification enzyme of a sandwich-type enzyme-linked immunosorbent assay (using horseradish peroxidase) with biotinylated dsDNA containing a T7 promoter, which allowed T7-mediated transcription of a Cas13a target, leading to its activation and to the collateral cleavage of a reporter.

\section{Outlook}

In less than five years, CRISPR-based diagnostics have evolved from an experimental nucleic acid sensing tool to a clinically relevant diagnostic technology for the fast, affordable and ultrasensitive sensing of biomarkers at the POC. However, there are several challenges that the technology needs to overcome to be able to deliver on the promise of transforming the way diseases are diagnosed and emerging pathogens monitored. A major shortcoming of most current CRISPR-based diagnostics is their dependence on preamplification for the detection of targets below the femtomolar range. Although the primers used for preamplification add another layer of specificity, this process adds complexity to the assay, increases its costs and prolongs the reaction time. The incorporation of non-primer-based signal-amplification strategies, or modifications of the Cas enzyme, crRNA or reporter molecule, are potential paths for improvement. Currently, most crRNAs and primers for preamplification have to be experimentally tested, and the design rules to narrow down potential candidates are coarse. Machine learning and bioinformatics algorithms based on large-scale experimental datasets could allow for the development of more effective design tools for the better prediction of crRNA performance and for improving crRNA specificity and activity. In fact, the feasibility of such approaches for crRNA design has been shown in the context of genome engineering ${ }^{102,103}$ and in the characterization and optimization of toehold switches ${ }^{104,105}$.

The ease of use of CRISPR-based diagnostics has been improved through optimized one-pot reactions and the simple visualization of test results. However, sample preparation still requires a separate step, and incubation temperatures higher than room temperature necessitate heating devices. In addition, target concentrations close to the LOD of the assay make it challenging to quantify the readout with certainty, especially when using the lateral-flow format. For usage at home or in the field, the assay design should combine simple sample-preparation protocols with robust detection methods to deliver robust results in variable or challenging contexts, such as prolonged storage, limited user training and harsh environmental conditions. To this end, devices that integrate sample preparation, sensing and reporting in single and easy-to-operate units are increasingly possible by using microfluidic techniques ${ }^{106}$. And the combination of such devices with digital analysis and data-sharing systems holds promise for patient-centric testing and remote data access. This is particularly important for mitigating the spread of infectious diseases by limiting unnecessary in-person contact in hospitals. Also, CRISPR-based diagnostics may enable patient-centric companion nucleic-acid-based diagnostics for monitoring treatment responses to a drug and to differentiate patients that are at risk for serious adverse effects from those that will probably benefit from the drug ${ }^{107}$. CRISPR-based diagnostics could facilitate the monitoring of genetic markers indicative of treatment response, such as mutations in the BRAF gene, which are commonly used to inform the treatment of melanoma skin cancer ${ }^{108}$. Furthermore, CRISPR-based diagnostics may be used for the real-time monitoring of gene expression across different tissues through the sensing of cell-free mRNA ${ }^{109}$.

Future applications of CRISPR-based diagnostics will probably involve technologies at the intersection with materials science ${ }^{79,80}$, for the sensing of nucleic acids on solid surfaces, on wearables and on medical devices. For example, ongoing work aims to incorporate SARS-CoV-2 sensors into face masks for the real-time 
detection of the virus. Direct sensing on surfaces can take advantage of high local concentrations of infectious agents and could allow for continuous tracking and for early diagnosis. This could extend the capabilities of monitoring strategies, such as the use of toilet systems for the analysis of excreta ${ }^{110}$.

Newly developed CRISPR assays must, of course, be validated ${ }^{83}$ by clinical trials, and assay validity monitored and maintained after clinical implementation. Yet, we believe that rapid innovation in CRISPR-based diagnostics will end up reshaping the technological landscape of nucleic-acid-based detection.

Received: 23 October 2020; Accepted: 2 June 2021; Published online: 16 July 2021

\section{References}

1. Neal, R. D. et al. Is increased time to diagnosis and treatment in symptomatic cancer associated with poorer outcomes? Systematic review. Br. J. Cancer https://doi.org/10.1038/bjc.2015.48 (2015).

2. Yang, S. \& Rothman, R. E. PCR-based diagnostics for infectious diseases: uses, limitations, and future applications in acute-care settings. Lancet Infect. Dis. https://doi.org/10.1016/S1473-3099(04)01044-8 (2004).

3. Weissleder, R., Lee, H., Ko, J. \& Pittet, M. J. COVID-19 diagnostics in context. Sci. Transl. Med. https://doi.org/10.1126/scitranslmed.abc193 (2020).

4. Burki, T. K. Testing for COVID-19. Lancet Respir. Med. https://doi. org/10.1016/S2213-2600(20)30247-2 (2020)

5. Mahony, J. B. et al. Cost analysis of multiplex PCR testing for diagnosing respiratory virus infections. J. Clin. Microbiol. https://doi.org/10.1128/ JCM.00556-09 (2009).

6. Wang, D. G., Brewster, J. D., Paul, M. \& Tomasula, P. M. Two methods for increased specificity and sensitivity in loop-mediated isothermal amplification. Molecules https://doi.org/10.3390/molecules20046048 (2015).

7. Mori, Y., Hirano, T. \& Notomi, T. Sequence specific visual detection of LAMP reactions by addition og cationic polymers. BMC Biotechnol. https:// doi.org/10.1186/1472-6750-6-3 (2006).

8. Phillips, E. A., Moehling, T. J., Bhadra, S., Ellington, A. D. \& Linnes, J. C. Strand displacement probes combined with isothermal nucleic acid amplification for instrument-free detection from complex samples. Anal. Chem. https://doi.org/10.1021/acs.analchem.8b00269 (2018).

9. De Baar, M. P. et al. One-tube real-time isothermal amplification assay to identify and distinguish human immunodeficiency virus type I subtypes A, $\mathrm{B}$, and $\mathrm{C}$ and circulating recombinant forms $\mathrm{AE}$ and ag. J. Clin. Microbiol. https://doi.org/10.1128/JCM.39.5.1895-1902.2001 (2001).

10. Woodford, N. \& Ellington, M. J. The emergence of antibiotic resistance by mutation. Clin. Microbiol. Infect. https://doi. org/10.1111/j.1469-0691.2006.01492.x (2007).

11. Irwin, K. K., Renzette, N., Kowalik, T. F. \& Jensen, J. D. Antiviral drug resistance as an adaptive process. Virus Evol. https://doi.org/10.1093/ve/ vew014 (2016)

12. Mojica, F. J. M., Díez-Villaseñor, C., García-Martínez, J. \& Soria, E. Intervening sequences of regularly spaced prokaryotic repeats derive from foreign genetic elements. J. Mol. Evol. https://doi.org/10.1007/s00239-0040046-3 (2005).

13. Makarova, K. S. et al. Evolution and classification of the CRISPR-Cas systems. Nat. Rev. Microbiol. https://doi.org/10.1038/nrmicro2577 (2011).

14. Bolotin, A., Quinquis, B., Sorokin, A. \& Dusko Ehrlich, S. Clustered regularly interspaced short palindrome repeats (CRISPRs) have spacers of extrachromosomal origin. Microbiology https://doi.org/10.1099/mic.0.28048 0 (2005).

15. Cong, L. et al. Multiplex genome engineering using CRISPR/Cas systems. Science https://doi.org/10.1126/science.1231143 (2013).

16. Hilton, I. B. et al. Epigenome editing by a CRISPR-Cas9-based acetyltransferase activates genes from promoters and enhancers. Nat. Biotechnol. https://doi.org/10.1038/nbt.3199 (2015).

17. Cox, D. B. T. et al. RNA editing with CRISPR-Cas13. Science https://doi org/10.1126/science.aaq0180 (2017)

18. Chen, B. et al. Dynamic imaging of genomic loci in living human cells by an optimized CRISPR/Cas system. Cell https://doi.org/10.1016/j. cell.2013.12.001 (2013)

19. Sheth, R. U., Yim, S. S., Wu, F. L. \& Wang, H. H. Multiplex recording of cellular events over time on CRISPR biological tape. Science https://doi. org/10.1126/science.aao0958 (2017).

20. Makarova, K. S. et al. An updated evolutionary classification of CRISPRCas systems. Nat. Rev. Microbiol. https://doi.org/10.1038/nrmicro3569 (2015)
21. Makarova, K. S. et al. Evolutionary classification of CRISPR-Cas systems: a burst of class 2 and derived variants. Nat. Rev. Microbiol. https://doi. org/10.1038/s41579-019-0299-x (2020).

22. Gootenberg, J. S. et al. Multiplexed and portable nucleic acid detection platform with Cas13, Cas12a and Csm6. Science 360, 439-444 (2018).

23. Santiago-Frangos, A. et al. Intrinsic signal amplification by type III CRISPR-Cas systems provides a sequence-specific SARS-CoV-2 diagnostic. Cell Reports Med. 2, 100319 (2021).

24. Zhang, Y. et al. Paired design of dCas9 as a systematic platform for the detection of featured nucleic acid sequences in pathogenic strains. ACS Synth. Biol. https://doi.org/10.1021/acssynbio.6b00215 (2017).

25. Pardee, K. et al. Rapid, low-cost detection of Zika virus using programmable biomolecular components. Cell 165, 1255-1266 (2016).

26. Bao, Y. et al. CUT-LAMP: contamination-free loop-mediated isothermal amplification based on the CRISPR/Cas9 cleavage. ACS Sens. https://doi. org/10.1021/acssensors.0c00034 (2020)

27. Zhou, W. et al. A CRISPR-Cas9-triggered strand displacement amplification method for ultrasensitive DNA detection. Nat. Commun. https://doi. org/10.1038/s41467-018-07324-5 (2018).

28. Compton, J. Nucleic acid sequence-based amplification. Nature https://doi. org/10.1038/350091a0 (1991).

29. Jiao, C. et al. Noncanonical crRNAs derived from host transcripts enable multiplexable RNA detection by Cas9. Science https://doi.org/10.1126/ science.abe7106 (2021)

30. East-Seletsky, A. et al. Two distinct RNase activities of CRISPR-C2c2 enable guide-RNA processing and RNA detection. Nature 538, 270-273 (2016).

31. Abudayyeh, O. O. et al. C2c2 is a single-component programmable RNA-guided RNA-targeting CRISPR effector. Science 353, aaf5573 (2016).

32. Gootenberg, J. S. et al. Nucleic acid detection with CRISPR-Cas13a/C2c2. Science 356, 438-442 (2017).

33. Piepenburg, O., Williams, C. H., Stemple, D. L. \& Armes, N. A. DNA detection using recombination proteins. PLoS Biol. https://doi.org/10.1371/ journal.pbio.0040204 (2006).

34. Kellner, M. J., Koob, J. G., Gootenberg, J. S., Abudayyeh, O. O. \& Zhang, F. SHERLOCK: nucleic acid detection with CRISPR nucleases. Nat. Protoc. https://doi.org/10.1038/s41596-019-0210-2 (2019).

35. Yan, W. X. et al. Functionally diverse type V CRISPR-Cas systems. Science https://doi.org/10.1126/science.aav7271 (2019).

36. Chen, J. S. et al. CRISPR-Cas12a target binding unleashes indiscriminate single-stranded DNase activity. Science https://doi.org/10.1126/science. aar6245 (2018).

37. Li, S.-Y. et al. CRISPR-Cas12a-assisted nucleic acid detection. Cell Discov. 4, 20 (2018).

38. Li, S. Y. et al. CRISPR-Cas12a has both cis- and trans-cleavage activities on single-stranded DNA. Cell Res. https://doi.org/10.1038/s41422-018-0022-x (2018).

39. Li, L. et al. HOLMESv2: a CRISPR-Cas12b-assisted platform for nucleic acid detection and DNA methylation quantitation. ACS Synth. Biol. 8, 2228-2237 (2019)

40. Notomi, T. et al. Loop-mediated isothermal amplification of DNA. Nucleic Acids Res. https://doi.org/10.1093/nar/28.12.e63 (2000).

41. Harrington, L. B. et al. Programmed DNA destruction by miniature CRISPR-Cas14 enzymes. Science https://doi.org/10.1126/science.aav4294 (2018).

42. Ramachandran, A. \& Santiago, J. G. CRISPR enzyme kinetics for molecular diagnostics. Anal. Chem. https://doi.org/10.1021/acs.analchem.1c00525 (2021).

43. Wölfel, R. et al. Virological assessment of hospitalized patients with COVID-2019. Nature https://doi.org/10.1038/s41586-020-2196-x (2020).

44. Fozouni, P. et al. Amplification-free detection of SARS-CoV-2 with CRISPR-Cas13a and mobile phone microscopy. Cell 184, 323-333 (2021)

45. Hajian, R. et al. Detection of unamplified target genes via CRISPR-Cas9 immobilized on a graphene field-effect transistor. Nat. Biomed. Eng. https:// doi.org/10.1038/s41551-019-0371-x (2019).

46. Bruch, R. et al. CRISPR/Cas13a-powered electrochemical microfluidic biosensor for nucleic acid amplification-free miRNA diagnostics. Adv. Mater. https://doi.org/10.1002/adma.201905311 (2019).

47. Niewoehner, O. et al. Type III CRISPR-Cas systems produce cyclic oligoadenylate second messengers. Nature https://doi.org/10.1038/ nature23467 (2017).

48. Kazlauskiene, M., Kostiuk, G., Venclovas, Č., Tamulaitis, G. \& Siksnys, V. A cyclic oligonucleotide signaling pathway in type III CRISPR-Cas systems. Science https://doi.org/10.1126/science.aao0100 (2017).

49. Moutsiopoulou, A. et al. Bioluminescent protein-inhibitor pair in the design of a molecular aptamer beacon biosensing system. Anal. Chem. https://doi. org/10.1021/acs.analchem.0c00518 (2020).

50. Storch, G. A. Diagnostic virology. Clin. Infect. Dis. https://doi. org $/ 10.1086 / 314015$ (2000) 
51. Asboe, D. et al. British HIV Association guidelines for the routine investigation and monitoring of adult HIV-1-infected individuals 2011. HIV Med. https://doi.org/10.1111/j.1468-1293.2011.00971.x (2012).

52. Pawlotsky, J. M. et al. EASL recommendations on treatment of hepatitis C 2018. J. Hepatol. https://doi.org/10.1016/j.jhep.2018.03.026 (2018).

53. Walker, G. T. et al. Strand displacement amplification-an isothermal, in vitro DNA amplification technique. Nucleic Acids Res. https://doi. org/10.1093/nar/20.7.1691 (1992)

54. Vincent, M., Xu, Y. \& Kong, H. Helicase-dependent isothermal DNA amplification. EMBO Rep. https://doi.org/10.1038/sj.embor.7400200 (2004).

55. Van Ness, J., Van Ness, L. K. \& Galas, D. J. Isothermal reactions for the amplification of oligonucleotides. Proc. Natl Acad. Sci. USA https://doi. org/10.1073/pnas.0730811100 (2003).

56. Lillis, L. et al. Factors influencing recombinase polymerase amplification (RPA) assay outcomes at point of care. Mol. Cell. Probes https://doi. org/10.1016/j.mcp.2016.01.009 (2016).

57. Watzinger, F., Ebner, K. \& Lion, T. Detection and monitoring of virus infections by real-time PCR. Mol. Aspects Med. https://doi.org/10.1016/j. mam.2005.12.001 (2006).

58. Whelan, J. A., Russell, N. B. \& Whelan, M. A. A method for the absolute quantification of cDNA using real-time PCR. J. Immunol. Methods https:// doi.org/10.1016/S0022-1759(03)00223-0 (2003).

59. Schmittgen, T. \& Livak, K. Analyzing real-time PCR data by the comparative CT method. Nat. Protoc. 3, 1101-1108 (2008).

60. Ding, $X$. et al. Ultrasensitive and visual detection of SARS-CoV-2 using all-in-one dual CRISPR-Cas12a assay. Nat. Commun. 11, 4711 (2020).

61. Lee, R. A. et al. Ultrasensitive CRISPR-based diagnostic for field-applicable detection of Plasmodium species in symptomatic and asymptomatic malaria Proc. Natl Acad. Sci. USA 117, 25722-25731 (2020).

62. Arizti-Sanz, J. et al. Streamlined inactivation, amplification, and Cas13-based detection of SARS-CoV-2. Nat. Commun. 11, 5921 (2020).

63. Joung, J. et al. Detection of SARS-CoV-2 with SHERLOCK one-pot testing. N. Engl. J. Med. https://doi.org/10.1056/nejmc2026172 (2020).

64. Nolan, T., Hands, R. E., Ogunkolade, W. \& Bustin, S. A. SPUD: A quantitative PCR assay for the detection of inhibitors in nucleic acid preparations. Anal. Biochem. https://doi.org/10.1016/j.ab.2006.01.051 (2006).

65. Smith, A. M. et al. Highly-multiplexed barcode sequencing: an efficient method for parallel analysis of pooled samples. Nucleic Acids Res. https:// doi.org/10.1093/nar/gkq368 (2010).

66. Elnifro, E. M., Ashshi, A. M., Cooper, R. J. \& Klapper, P. E. Multiplex PCR: optimization and application in diagnostic virology. Clini. Microbiol. Rev. https://doi.org/10.1128/CMR.13.4.559-570.2000 (2000).

67. Ackerman, C. M. et al. Massively multiplexed nucleic acid detection with Cas13. Nature 582, 277-282 (2020).

68. Gilpatrick, T. et al. Targeted nanopore sequencing with Cas9-guided adapter ligation. Nat. Biotechnol. https://doi.org/10.1038/s41587-020-0407-5 (2020).

69. Giesselmann, P. et al. Analysis of short tandem repeat expansions and their methylation state with nanopore sequencing. Nat. Biotechnol. https://doi. org/10.1038/s41587-019-0293-x (2019).

70. Gu, W. et al. Depletion of abundant sequences by hybridization (DASH): using Cas9 to remove unwanted high-abundance species in sequencing libraries and molecular counting applications. Genome Biol. https://doi. org/10.1186/s13059-016-0904-5 (2016).

71. Quan, J. et al. FLASH: a next-generation CRISPR diagnostic for multiplexed detection of antimicrobial resistance sequences. Nucleic Acids Res. https:// doi.org/10.1093/nar/gkz418 (2019).

72. Katzmeier, F. et al. A low-cost fluorescence reader for in vitro transcription and nucleic acid detection with Cas13a. PLoS ONE https://doi.org/10.1371/ journal.pone.0220091 (2019).

73. Yuan, C. et al. Universal and naked-eye gene detection platform based on the clustered regularly interspaced short palindromic repeats/Cas12a/13a system. Anal. Chem. https://doi.org/10.1021/acs.analchem.9b05597 (2020).

74. $\mathrm{Hu}, \mathrm{M}$. et al. Single-step, salt-aging-free, and thiol-free freezing construction of AuNP-based bioprobes for advancing CRISPR-based diagnostics. J. Am. Chem. Soc. https://doi.org/10.1021/jacs.0c00217 (2020).

75. Spoelstra, W. K. et al. CRISPR-based DNA and RNA detection with liquid-liquid phase separation. Biophys. J. 120, 1198-1209 (2021).

76. Wang, X. et al. Clustered regularly interspaced short palindromic repeats/ Cas9-mediated lateral flow nucleic acid assay. ACS Nano https://doi. org/10.1021/acsnano.0c00022 (2020).

77. Dai, Y. et al. Exploring the trans-cleavage activity of CRISPR-Cas12a (cpf1) for the development of a universal electrochemical biosensor. Angew. Chem. Int. Ed. 58, 17399-17405 (2019).

78. Zhou, T. et al. CRISPR/Cas13a powered portable electrochemiluminescence chip for ultrasensitive and specific MiRNA detection. Adv. Sci. https://doi. org/10.1002/advs.201903661 (2020).

79. English, M. A. et al. Programmable CRISPR-responsive smart materials. Science https://doi.org/10.1126/science.aaw5122 (2019).
80. Gayet, R. V. et al. Creating CRISPR-responsive smart materials for diagnostics and programmable cargo release. Nat. Protoc. https://doi. org/10.1038/s41596-020-0367-8 (2020).

81. Myhrvold, C. et al. Field-deployable viral diagnostics using CRISPR-Cas13. Science 360, 444-448 (2018).

82. Ladha, A., Joung, J., Abudayyeh, O. O., Gootenberg, J. S. \& Zhang, F. A 5-min RNA preparation method for COVID-19 detection with RT-qPCR. Preprint at medRxiv https://doi.org/10.1101/2020.05.07.20055947 (2020).

83. Patchsung, M. et al. Clinical validation of a Cas13-based assay for the detection of SARS-CoV-2 RNA. Nat. Biomed. Eng. https://doi.org/10.1038/ s41551-020-00603-x (2020).

84. Kaminski, M. M. et al. A CRISPR-based assay for the detection of opportunistic infections post-transplantation and for the monitoring of transplant rejection. Nat. Biomed. Eng. 4, 601-609 (2020).

85. Qin, P. et al. Rapid and fully microfluidic Ebola virus detection with CRISPR-Cas13a. ACS Sens. https://doi.org/10.1021/acssensors.9b00239 (2019).

86. Zhang, F., Abudayyeh, O. O., Gootenberg, J. S., Sciences, C. \& Mathers, L. A protocol for detection of COVID-19 using CRISPR diagnostics. Bioarchive (2020).

87. Broughton, J. P. et al. CRISPR-Cas12-based detection of SARS-CoV-2. Nat. Biotechnol. https://doi.org/10.1038/s41587-020-0513-4 (2020).

88. Wu, Y., Liu, S. X., Wang, F. \& Zeng, M. S. Room temperature detection of plasma Epstein-Barr virus DNA with CRISPR-Cas13. Clin. Chem. https:// doi.org/10.1373/clinchem.2018.299347 (2019).

89. Ai, J. W. et al. CRISPR-based rapid and ultra-sensitive diagnostic test for Mycobacterium tuberculosis. Emerg. Microbes Infect. 8, 1361-1369 (2019).

90. Guk, K. et al. A facile, rapid and sensitive detection of MRSA using a CRISPR-mediated DNA FISH method, antibody-like dCas9/sgRNA complex. Biosens. Bioelectron. https://doi.org/10.1016/j.bios.2017.04.016 (2017).

91. Huang, M., Zhou, X., Wang, H. \& Xing, D. Clustered regularly interspaced short palindromic repeats/Cas9 triggered isothermal amplification for site-specific nucleic acid detection. Anal. Chem. https://doi.org/10.1021/acs. analchem.7b04542 (2018).

92. Shen, J., Zhou, X. \& Shan, Y. et al. Sensitive detection of a bacterial pathogen using allosteric probe-initiated catalysis and CRISPR-Cas13a amplification reaction. Nat. Commun. 11, 267 (2020).

93. Cunningham, C. H. et al. A novel CRISPR-based malaria diagnostic capable of Plasmodium detection, species differentiation, and drug-resistance genotyping. EBioMedicine 68, 103415 (2021).

94. Shan, Y., Zhou, X., Huang, R. \& Xing, D. High-fidelity and rapid quantification of miRNA combining crRNA programmability and CRISPR/ Cas13a trans-cleavage activity. Anal. Chem. https://doi.org/10.1021/acs. analchem.9b00073 (2019).

95. Wang, G., Tian, W., Liu, X., Ren, W. \& Liu, C. New CRISPR-derived microRNA sensing mechanism based on Cas12a self-powered and rolling circle transcription-unleashed real-time crRNA recruiting. Anal. Chem. https://doi.org/10.1021/acs.analchem.0c00680 (2020).

96. SARS-CoV-2 variant classifications and definitions. CDC https://www.cdc gov/coronavirus/2019-ncov/variants/variant-info.html (2021).

97. Liang, M. et al. A CRISPR-Cas12a-derived biosensing platform for the highly sensitive detection of diverse small molecules. Nat. Commun. 10, 3672 (2019).

98. Iwasaki, R. S. \& Batey, R. T. SPRINT: a Cas13a-based platform for detection of small molecules. Nucleic Acids Res. https://doi.org/10.1093/nar/gkaa673 (2020).

99. Peng, L. et al. CRISPR-Cas12a based aptasensor for sensitive and selective ATP detection. Sens. Actuators B https://doi.org/10.1016/j.snb.2020.128164 (2020).

100. Xiong, Y. et al. Functional DNA regulated CRISPR-Cas12a sensors for point-of-care diagnostics of non-nucleic-acid targets. J. Am. Chem. Soc. https://doi.org/10.1021/jacs.9b09211 (2020).

101. Chen, Q., Tian, T., Xiong, E., Wang, P. \& Zhou, X. CRISPR/Cas13a signal amplification linked immunosorbent assay for femtomolar protein detection. Anal. Chem. https://doi.org/10.1021/acs.analchem.9b04403 (2020).

102. Kim, H. K. et al. SpCas9 activity prediction by DeepSpCas9, a deep learning-based model with high generalization performance. Sci. Adv. https://doi.org/10.1126/sciadv.aax9249 (2019).

103. Chuai, G. et al. DeepCRISPR: optimized CRISPR guide RNA design by deep learning. Genome Biol. https://doi.org/10.1186/s13059-018-1459-4 (2018).

104. Valeri, J. A. et al. Sequence-to-function deep learning frameworks for engineered riboregulators. Nat. Commun. https://doi.org/10.1038/ s41467-020-18676-2 (2020).

105. Angenent-Mari, N. M., Garruss, A. S., Soenksen, L. R., Church, G. \& Collins, J. J. A deep learning approach to programmable RNA switches. Nat. Commun. https://doi.org/10.1038/s41467-020-18677-1 (2020).

106. Shao, N., Han, X., Song, Y., Zhang, P. \& Qin, L. CRISPR-Cas12a coupled with platinum nanoreporter for visual quantification of SNVs on a volumetric bar-chart chip. Anal. Chem. https://doi.org/10.1021/acs. analchem.9b02925 (2019). 
107. In vitro companion diagnostic devices. US Food and Drug Administration http://www.fda.gov/regulatory-information/search-fda-guidance-documents/ vitro-companion-diagnostic-devices (2019).

108. Cheng, L., Lopez-Beltran, A., Massari, F., Maclennan, G. T. \& Montironi, R Molecular testing for BRAF mutations to inform melanoma treatment decisions: a move toward precision medicine. Mod. Pathol. https://doi org/10.1038/modpathol.2017.104 (2018).

109. Koh, W. et al. Noninvasive in vivo monitoring of tissue-specific global gene expression in humans. Proc. Natl Acad. Sci. USA https://doi.org/10.1073/ pnas.1405528111 (2014).

110. Park, S.-m. et al. A mountable toilet system for personalized health monitoring via the analysis of excreta. Nat. Biomed. Eng. https://doi. org/10.1038/s41551-020-0534-9 (2020).

111. Wang, T., Liu, Y., Sun, H. H., Yin, B. C. \& Ye, B. C. An RNA-guided Cas9 nickase-based method for universal isothermal DNA amplification. Angew. Chem. Int. Ed. https://doi.org/10.1002/anie.201901292 (2019).

112. Teng, F. et al. CDetection: CRISPR-Cas12b-based DNA detection with sub-attomolar sensitivity and single-base specificity. Genome Biol. 20, 132 (2019).

\section{Acknowledgements}

M.M.K. was supported by the Emmy Noether Programme (KA5060/1-1) of the German Research Foundation (DFG). J.J.C. was supported by the Paul G. Allen Frontiers Group and the Wyss Institute. All figures were created with BioRender.com.

\section{Author contributions}

All authors contributed to writing and revising the manuscript, and approved the final version.

\section{Competing interests}

J.J.C. is a co-founder and director of Sherlock Biosciences. O.O.A., J.S.G. and F.Z. are co-founders and scientific advisors for Sherlock Biosciences and Pine Trees Health. O.O.A. and J.S.G. are also co-founders and scientific advisors for Moment Biosciences and Tome Biosciences. F.Z. is also a co-founder of Editas Medicine, Beam Therapeutics, Pairwise Plants and Arbor Biotechnologies.

\section{Additional information}

Correspondence should be addressed to J.J.C.

Peer review information Nature Biomedical Engineering thanks Kiana Aran, Charles Chiu and Da Xing for their contribution to the peer review of this work.

Reprints and permissions information is available at www.nature.com/reprints. Publisher's note Springer Nature remains neutral with regard to jurisdictional claims in published maps and institutional affiliations.

(C) Springer Nature Limited 2021 